\title{
Soft materials for wearable supercapacitors
}

\author{
Lili Jiang, Le Yuan, Wei Wang, Qinyong Zhang \\ Key Laboratory of Fluid and Power Machinery of Ministry of Education, School of Materials Science and Engineering, Xihua \\ University, Chengdu 610039, Sichuan, China. \\ Correspondence to: Prof. Lili Jiang, Key Laboratory of Fluid and Power Machinery of Ministry of Education, School of Materials \\ Science and Engineering, Xihua University, 999\# Jinzhou Road, Jinniu district, Chengdu 610039, Sichuan, China. \\ E-mail: qvinjiang@163.com
}

How to cite this article: Jiang L, Yuan L, Wang W, Zhang Q. Soft materials for wearable supercapacitors. Soft Sci 2021;1:5. https://dx.doi.org/10.20517/ss.2021.07

Received: 21 Jul 2021 First Decision: 28 Jul 2020 Revised: 4 Aug 2021 Accepted: 7 Aug 2021 First online: 7 Aug 2021

Academic Editor: Zhifeng Ren Copy Editor: Yue-Yue Zhang Production Editor: Yue-Yue Zhang

\begin{abstract}
Along with the rapid progress of wearable and portable electronic devices including electrical sensors, flexible displays, and health monitors, there is an ever-growing demand for wearable power sources. Supercapacitors, as a new kind of energy storage device, have received considerable attention for decades due to their high power density, excellent cycling stability, and easy fabrication. To fulfill the demand of wearable power sources, wearable supercapacitors are also further developed and studied. New electrode materials that play a significant role in determining both the wearability and electrochemical performance of wearable supercapacitors are also extensively explored. Herein, the recent progress on wearable soft electrode/electrolyte materials and the structure design strategies for developing wearable supercapacitors are summarized. Additionally, the existing challenges in current technologies and research are highlighted and discussed with the hope of inspiring future studies.
\end{abstract}

Keywords: Soft materials, electrodes, electrolytes, structure design, wearable supercapacitors

\section{INTRODUCTION}

Nowadays, along with the rapid growth of the electronic industry, portable/wearable electronics including health monitors, electronic sensors, and human healthcare equipments are becoming a noticeable technological trend that has experienced rapid growth ${ }^{[1,2]}$. In this regard, wearable energy storage devices are obviously indispensable parts for portable/wearable electronics ${ }^{[3-5]}$. The design and fabrication of the power

The Author(s) 2021. Open Access This article is licensed under a Creative Commons Attribution 4.0 International License (https://creativecommons.org/licenses/by/4.0/), which permits unrestricted use, sharing, adaptation, distribution and reproduction in any medium or format, for any purpose, even commercially, as long as you give appropriate credit to the original author(s) and the source, provide a link to the Creative Commons license, and indicate if changes were made. 
supply systems with high flexibility and high energy and power densities is needed in current rechargeable energy storage markets. The most applied, Li-ion batteries are not very suitable for portable/wearable electronics due to the issues of rigid and bulky electrode and the toxic $\mathrm{Li}^{+}$electrolyte ${ }^{[2]}$. Additionally, studies also indicated that the heat generated from the commercially available Li-ion batteries would affect biologic tissues and limit their application in portable/wearable electronics ${ }^{[6,7]}$.

In this context, high-performance and functional supercapacitors (SCs), as a kind of newly developed energy storage device, have already been extensively applied to meet the pressing demand for future wearable electronics due to their long-term stability, rapid charge - discharge capability, and temperature tolerance ${ }^{[8,9]}$. To further fulfill the specific energy demands of the aforementioned external and in vivo portable electronics, wearable supercapacitors (WSCs) that can be stretched, compressed, bent, twisted, and deformed into arbitrary shapes provide a promising alternative. However, on the one hand, most commercial SCs are fully or partially composed of planar and rigid materials, which require the use of obtrusive, hard supports and easily cause discomfort and instable power output. On the other hand, the electrolytes for commercial SCs are most commonly aqueous electrolytes including salt, acid, and alkaline solutions, which are toxic when used in external or in vivo wearable electronic ${ }^{[1,111]}$. Thus, the replacement of the bulky, rigid, and toxic materials is needed. The enhancement of the wearability and functionality of SCs is also significant to accelerate the investigation and design of WSCs.

WSCs for external or in vivo wearable electronics should provide user comfort, compliant mechanics, soft integration, multifunctionality, and, especially, the stable and intimate contact to the soft human tissues without adding any mechanical and thermal loading or causing tissue breakdown. Therefore, advanced soft materials that are designed or fabricated for the utilization of electrodes and electrolytes of WSCs are extremely desirable and significant. Tremendous research efforts have been directed at the design of advanced soft electrode materials and electrolytes for WSCs that could partly address the aforementioned functions of WSCs.

This review summarizes the recent progress in WSCs with flexible, stretchable, and textile characteristics. We discuss the most widely applied electrode materials (graphene, carbon nanotubes, conductive polymers, metal compounds, etc.) for WSCs, the newly developed electrode materials (hydrogels, MOFs, and MXenes), and solid-state electrolytes for WSCs with an emphasis on the configuration, design principles, and electrochemical performance [Figure 1 $]^{[12-17]}$. Additionally, the prospects and challenges for the development of WSCs for wearable and implantable electronic devices are highlighted and discussed, with the aim of inspiring further research and development in the field of bioelectronics.

\section{STRUCTURAL CHARACTERISTICS AND ENERGY STORAGE PRINCIPLES OF SCS}

SCs have attracted notable scientific attention in the past decades due to their potential of clean energy, easy assembly, and high performance. The advantageous characteristics of SCs have also contributed to the rapid growth of low-power electronics, including wearable and portable electronic devices ${ }^{[1,1,1]}$. Figure $2 \mathrm{~A}$ schematically illustrates the structure and energy storage mechanisms of supercapacitors: (1) Electrochemical double layer capacitance (EDLC) presents the immediate formation of an electrical double layer on the surface of the electrodes [Figure 2B]. EDLCs exhibit higher energy densities than conventional capacitors with their high effective surface space and minimal charge separation distances. (2) Pseudocapacitance presents fast reversible redox reactions or reversible intercalation on the surface of the active electrode materials. Reversible redox chemical reactions combine with dynamic equilibrium oxidation, the adsorption and desorption of ions on the surface of electrochemical active materials [Figure $2 \mathrm{C}$ ], while the reversible intercalation and exfoliation processes move ions in the electrolyte between 


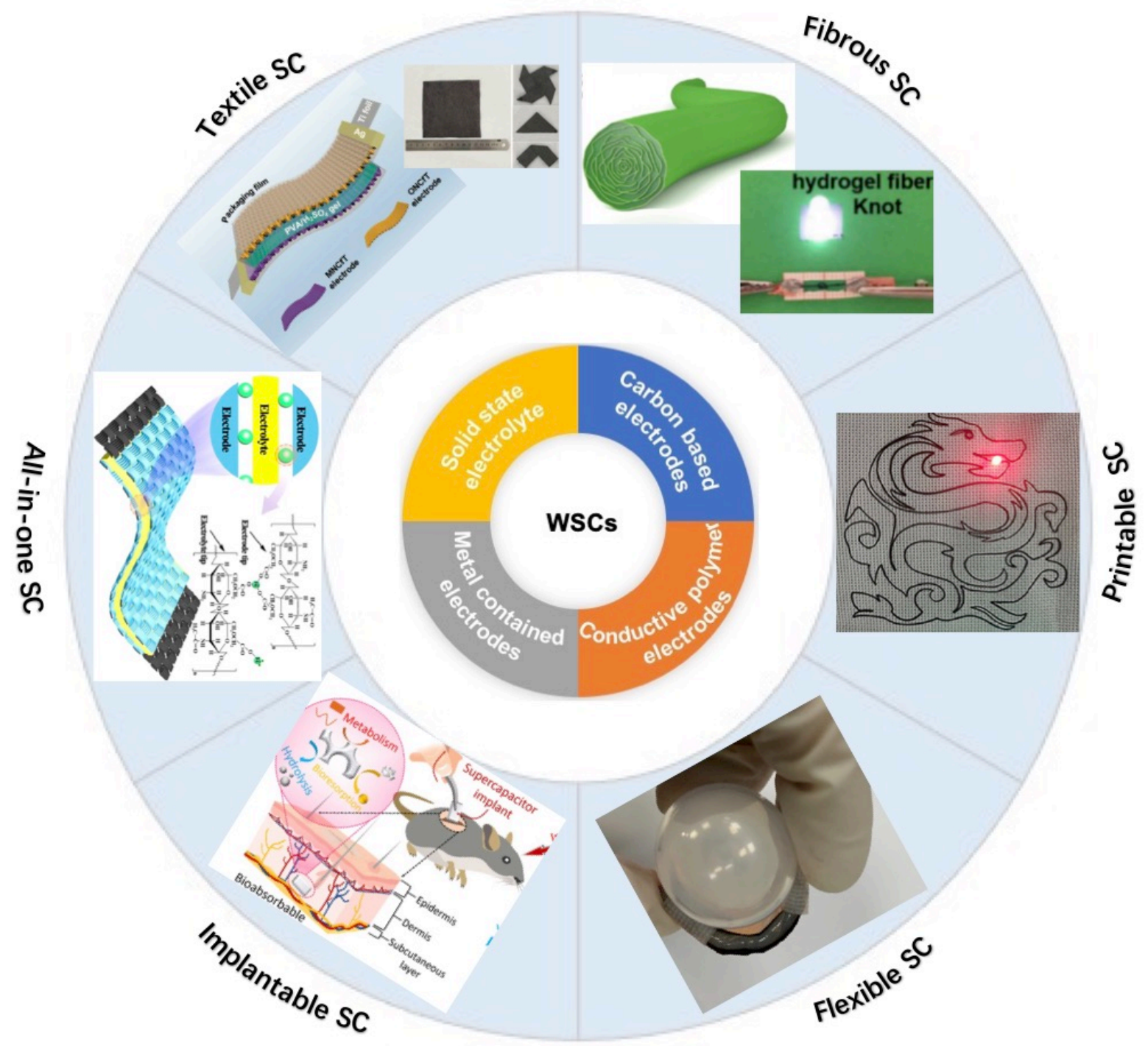

Figure 1. A graphic overview of this review. Section 2 introduces the structural characteristics and energy storage principles of SCs. Section 3 discusses the required properties of soft materials for WSCs. Section 4 presents soft electrode materials for wearable supercapacitors. Section 5 introduces studies on solid-state electrolytes for WSCs. Section 6 introduces recent studies on all-in-one wearable supercapacitor devices. The photographs on top are reproduced with permission ${ }^{[12-17]}$. WSCs: Wearable supercapacitors; $\mathrm{SCs}$ supercapacitors.

the electrodes during the electrochemical energy storage process ${ }^{[20-22]}$ [Figure 2D].

According to the above-mentioned energy storage mechanisms, the performance of SCs is primarily dependent on the electrode materials and the interactions between the electrode and the electrolyte. The electrochemical properties, conductivity, and specific surface areas of the electrode materials, as well as the ion conductivity of the electrolyte, are all key factors that determine the electrochemical performance of SCs, which is usually reflected by capacitance, energy density, and power density. For capacitance, including gravimetric specific capacitance $\left(\mathrm{F} \mathrm{g}^{-1}\right)$, area capacitance $\left(\mathrm{F} \mathrm{cm}^{-2}\right)$, and volume capacitance $\left(\mathrm{F} \mathrm{cm}^{-3}\right)$, which are chosen depending on the shape or structure of the SCs, can be calculated from the cyclic voltammetry (CV) or the galvanostatic charge-discharge data. For energy density and power density of SCs, their units depend on the unit of capacitance. Equations (1)-(3) are taken as examples to calculate the gravimetric 


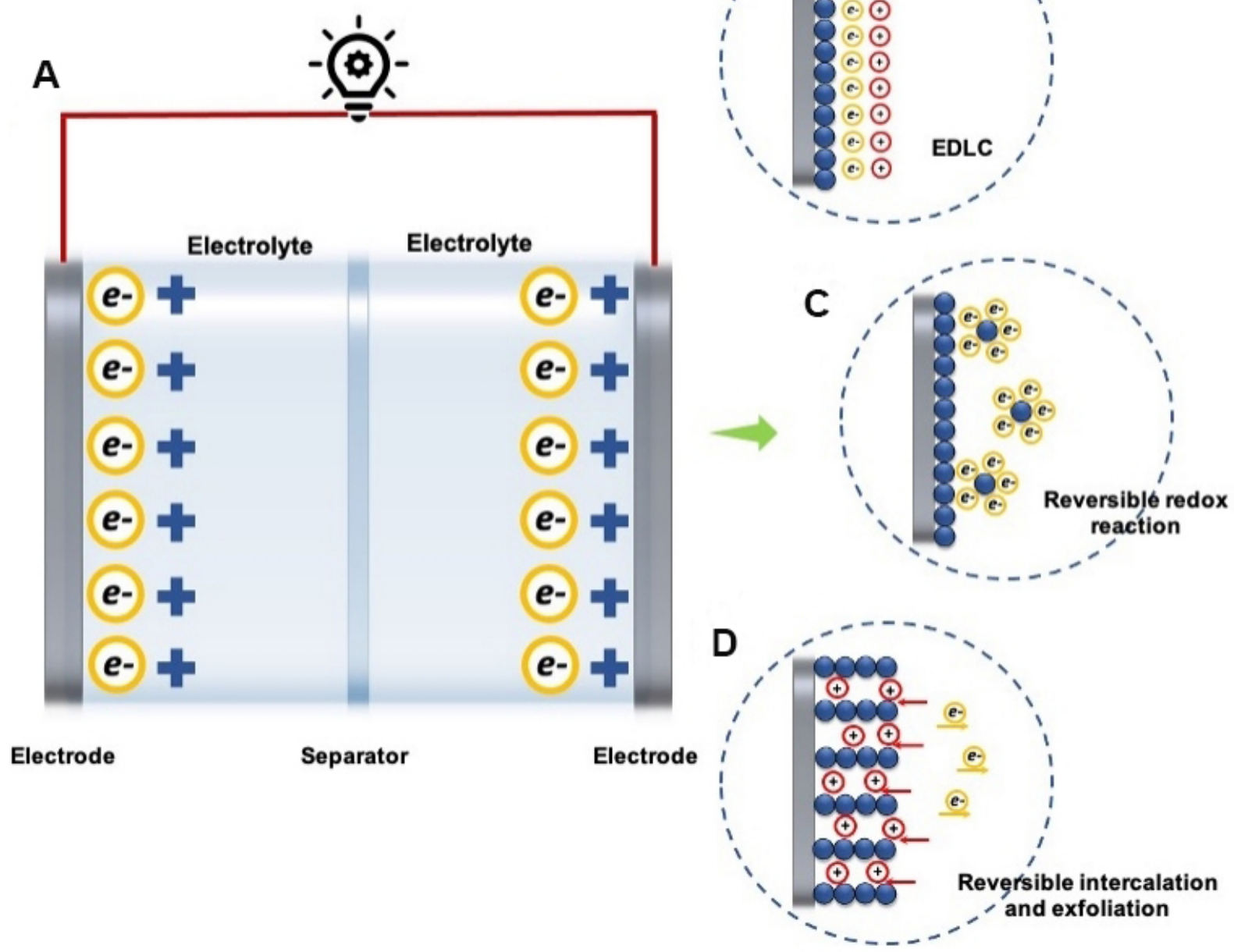

Figure 2. (A) Schematic structure of a supercapacitor. Energy storage mechanisms illustration: (B) EDLC; (C) reversible redox reaction; and $(D)$ reversible intercalation and exfoliation process. EDLC: Electrochemical double layer capacitance.

specific capacitance $\left[\mathrm{C}_{\mathrm{s}}\right.$, Equation (1)], energy density $\left[\mathrm{E}_{\mathrm{s}}\right.$, Equation (2)], and power density $\left[\mathrm{P}_{\mathrm{s}}\right.$, Equation (3)] based on CV data.

$$
\begin{aligned}
& C_{s}=\frac{\int I d v}{v m \Delta V} \\
& E_{s}=\frac{1}{m} \int I V(t) d t \\
& P_{s}=\frac{E_{s}}{t}
\end{aligned}
$$


where $I$ is the constant current, $m$ is the total mass of the active materials, $v$ is the scan rate, and $\Delta \mathrm{V}$ is the width of the voltage window ${ }^{[17]}$.

Numerous attempts have been made to improve the electrochemical performance of SCs through these $\operatorname{aspects}^{[23-28]}$. Moreover, considering the structural characteristics of SCs, achieving advanced multifunctional WSCs depend on the specific characteristics of the electrode materials. Meanwhile, replacing the aqueous electrolytes in conventional SCs is also significant for preparing WSCs for wearable and portable electronics.

\section{REQUIRED PROPERTIES OF SOFT MATERIALS FOR WSCS}

To further meet the requirements of external/in vivo WSCs, both the electrode materials and electrolyte materials need to be deformed into arbitrary shapes to adapt to the human physiological and musculoskeletal environment. Conventional brittle and rigid electrode materials could result in fractures when accommodating a small amount of imposed strain, which is inappropriate for fully stretchable devices. Thus, some special properties, different from those of traditional bulk and rigid materials, are highly needed for SCs.

Table 1 summarizes the required properties of electrode materials for WSCs. Young's modulus is a mechanical property that measures the tensile stiffness of a solid material. It quantifies the relationship between tensile stress and strain [(proportional deformation) in the linear elastic reign of a material Figure $3 \mathrm{~A}]^{[2,3,30]}$. Corresponding to the Young's modulus value of the skin or other human soft tissues, a small value $\left(10^{1}-10^{6} \mathrm{kP}\right)$ in a broad stress range is required for WSCs ${ }^{[31]}$, However, the most used electrode materials including carbon and metal oxides exhibit higher Young's modulus values, as shown in Figure 3B. To address this issue, downscaling the electrically active materials and embedding them in or onto soft materials that have lower Young's modulus values is one of the most popular strategies to fabricate $\mathrm{WSCs}^{[32,33]}$. Stretchability is also significant for WSCs because biological tissues are capable of enduring high dynamic and mechanical stress. For example, the skin, muscles, and peripheral nerves can experience $30 \%$ tensile strain and displacement during exercise in conventional postures ${ }^{[34,35]}$. Thus, high stretchability is needed for soft materials for WSCs which could be deformed into arbitrary shapes. Similar to the case of Young's modulus performance, the normally used electrode materials for SCs possess low stretchability [Figure $3 \mathrm{C}$ ], which could also be improved by downscaling the dimension of active materials or embedding them in or onto soft materials with higher stretchability ${ }^{[36-39]}$. Conductivity is also an essential property, which determines the electrochemical performance of SCs, especially the cycle stability and rate performance. As shown in Figure 3D, active materials with low Young's modulus and high stretchability usually possess low conductivity. Therefore, many studies have been committed to improving the conductivity through doping or combining materials with high conductivity ${ }^{[17,40-43]}$. In addition, due to the direct contact with human skin, active materials with high biocompatibility are also desired, especially for implantable SCs ${ }^{[44-46]}$. According to these requirements, the following sections summarize conventional "soft" electrode materials and recently developed "soft" electrode materials for WSCs, from the aspects of material properties and preparation strategies.

\section{SOFT ELECTRODE MATERIALS FOR WEARABLE SUPERCAPACITORS}

According to the storage mechanism of SCs discussed above, the most significant part that determines the electrochemical property of SCs is the electrode material, which is also the key part to meet the demands of wearable electronics. In the following, conventional electrode materials including carbon-based electrodes, polymer-based soft electrodes, and metal-containing soft composites and their specific preparation strategies are summarized and discussed. Moreover, some newly developed soft electrode materials are also highlighted. 
Table 1. Necessary mechanical and physicochemical properties for WSCs

\begin{tabular}{|c|c|c|c|c|}
\hline Properties & Description & Typical strategies & Proper values & Ref. \\
\hline $\begin{array}{l}\text { Young's } \\
\text { modulus }\end{array}$ & $\begin{array}{l}\text { Proper value is required for direct contact with } \\
\text { human skin or implantable SCs }\end{array}$ & $\begin{array}{l}\text { Embedding inorganics in/onto the organic } \\
\text { substrate/hydrogels }\end{array}$ & $10^{1}-10^{6} \mathrm{kP}$ & {$[32,33]$} \\
\hline \multirow[t]{3}{*}{ Stretchability } & \multirow[t]{3}{*}{$\begin{array}{l}\text { Essential when being deformed into arbitrary } \\
\text { shapes }\end{array}$} & $\begin{array}{l}\text { Downscaling the dimension of active } \\
\text { materials/electrode materials (OD, 1D, 2D) }\end{array}$ & & \multirow[t]{3}{*}{ [36-39] } \\
\hline & & Being printed onto plastic substrates & Higher than $10^{\circ}$ & \\
\hline & & Strain-tolerant electrode/collector & & \\
\hline \multirow[t]{3}{*}{ Conductivity } & \multirow{3}{*}{$\begin{array}{l}\text { High conductivity is required for high cycle stability } \\
\text { and rate performance }\end{array}$} & Carbon-based composite materials & & \multirow{3}{*}{$\begin{array}{l}{[17,40-} \\
43]\end{array}$} \\
\hline & & Bimetal oxides & & \\
\hline & & $\begin{array}{l}\text { Metal compounds (metal nitrides, metal } \\
\text { sulfides, etc.) } \\
\text { Conductive metal-organic frameworks } \\
\text { (MOFs) } \\
\text { MXene }\end{array}$ & $\begin{array}{l}\text { Higher than } \\
10^{-2}\left(\mathrm{~S} \mathrm{~m}^{-1}\right)\end{array}$ & \\
\hline \multirow[t]{3}{*}{ Biocompatibility } & \multirow{3}{*}{$\begin{array}{l}\text { Low toxicity or nontoxicity is required for long-term } \\
\text { retention of WSCs on human skin or in the human } \\
\text { body }\end{array}$} & $\begin{array}{l}\text { Encapsulation or compositing with } \\
\text { biocompatible materials }\end{array}$ & $\begin{array}{l}\text { No toxicity to } \\
\text { cells or tissues }\end{array}$ & \multirow[t]{3}{*}{ [44-46] } \\
\hline & & $\begin{array}{l}\text { Adopting natural or intrinsically nontoxic } \\
\text { polymers and metal oxides }\end{array}$ & & \\
\hline & & Biodegradable materials & & \\
\hline
\end{tabular}

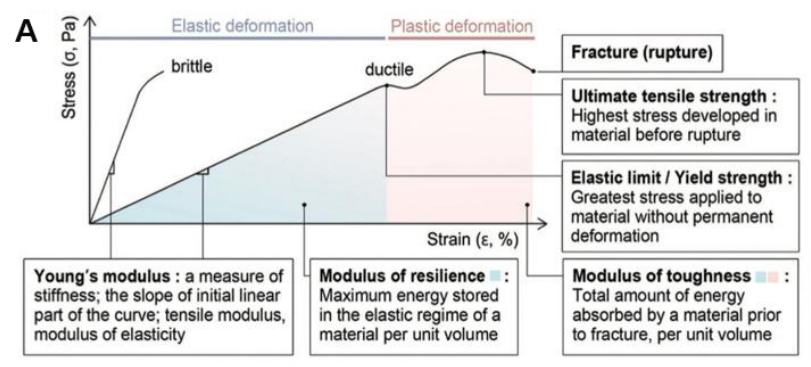

C

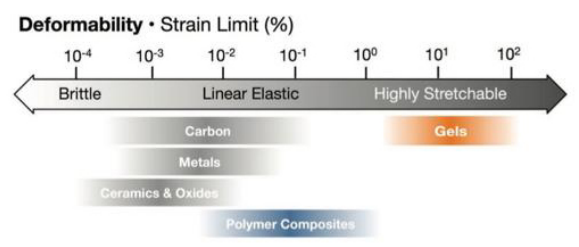

B

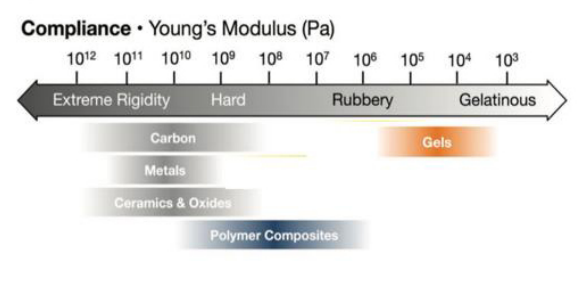

D

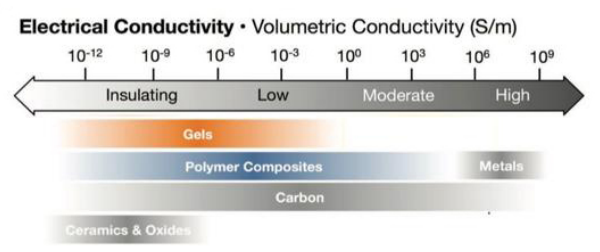

Figure 3. Summarized required properties for WSCs. (A) Stress-strain profiles of brittle and ductile materials and the description of important material properties ${ }^{[29]}$. (B-D) The compliance, deformability, and electrical conductivity of widely used materials for WSCs, respectively ${ }^{[30]}$.WSCs: Wearable supercapacitors.

\section{Carbon-based soft electrodes for wearable supercapacitors}

As the most developed electrode materials, carbon-based materials with EDLC behavior have attracted huge attention as electrode materials for SCs. The mostly widely used are active carbons or porous carbons derived from natural mass (e.g., wood ${ }^{[47]}$, coal ${ }^{[48]}$, nutshell ${ }^{[49]}$, loofah ${ }^{[50]}$, etc.) by physical or chemical process, which are not available for WSCs due to their bulkiness and brittleness. Thus, according to Figure 3 and Table 1, to further adapt them to the development of WSCs, down scaling the dimension, embedding them on or into organic substrates/hydrogels, and specific structures would endow them with the appropriate material properties (low Young's modulus, high deformability, and high conductivity) as soft electrodes for WSCs. 


\section{Graphene-based electrodes for WSCs}

Graphene has already been widely studied as an electrode for SCs due to its excellent electrical, mechanical, and capacitance properties ${ }^{[1,52]}$. Furthermore, its easy assembling onto different soft substrates property makes it a promising candidate electrode for WSCs. In this section, recent advanced research on graphenebased soft electrode for WSCs is reviewed.

\section{Flexible and free-standing graphene-based electrodes}

Graphene can be easily assembled into mechanically strong films due to its two-dimensional (2D) structure and stacking trend ${ }^{[53]}$. Thus, flexible and free-standing graphene-based electrodes have been wildly prepared as electrodes for flexible SCs. Reduced graphene oxide ( $\mathrm{rGO}$ ) produced by chemical redox process is the most widely used electrodes for SCs due to its lower production cost, and it can be easily modified and assembled into strong films or onto different flexible substrates ${ }^{[44,55]}$.

Vacuum filtrating and chemical reduction process were first used to prepared rGO papers, which possess a Young's modulus of $41.8 \mathrm{GPa}$, a tensile strength of $293.3 \mathrm{MPa}$, and an electrical conductivity of $118 \mathrm{~S}$ $\mathrm{cm}^{-1[56]}$. This study showed the feasibility of rGO to be applied as a free-standing and flexible electrode for SCs. Freeze-drying processes were also applied to prepare free-standing and flexible rGO papers ${ }^{[57]}$. The obtained rGO electrode also possessed good conductivity of $18 \mathrm{~S} \mathrm{~cm}^{-1}$ and capacitance of $172 \mathrm{~F} \mathrm{~g}^{-1}$ at current density of $1 \mathrm{~A} \mathrm{~g}^{-1}$ capacitance. These results prove the potential application of $\mathrm{rGO}$ paper-like electrode materials for WSCs. To further improve their electrochemical performance, strategies also focus on improving the conductivity of these rGO papers when used as electrodes for WSCs ${ }^{[58]}$. However, the tightly stacked nature of free-standing rGO films lowers the electrolyte accessible surface area and blocks the formation of electrical double layers, leading to lower electrochemical capacitance performance.

To address this issue, the most common strategy is to introduce a porous structure into rGO layers, which can effectively increase the electrolyte accessible surface area and improve the electrochemical performance of flexible and free-standing paper-like graphene electrodes. One typical porous structure is graphene hydrogels/aerogel ${ }^{[59-62]}$. These studies proved that this interconnected porous structure could improve the electrochemical performance of graphene-based electrodes by lower rGO sheet stacking, more accessible specific surface area, and rapid ion diffusion and electron transport throughout the entire interconnected porous network. Moreover, according to the energy storage principle (EDLC and pseudocapacitance) of SCs, the introduction of pseudocapacitive components into rGO sheets during the preparation process can not only hinder the re-stacking of rGO sheets but also further improve either the mechanical or electrochemical properties of as-prepared free-standing and flexible electrodes for WSCs ${ }^{[63-67]}$. Our groups prepared ternary composite free-standing and flexible rGO-based films. The vaporing process was carried out to firstly produce free-standing and flexible $\mathrm{rGO}-\mathrm{TiO}_{2}$ films. $\mathrm{TiO}_{2}$ nanoparticles were introduced to hinder the stacking of rGO sheets and contribute their pseudocapacitance. To further improve the capacitance performance while still keeping the flexibility of rGO films, conductive polymers (CPs) were deposited on the surface of $\mathrm{rGO}-\mathrm{TiO}_{2}$ films to form ternary free-standing and flexible $\mathrm{rGO}-\mathrm{TiO}_{2}-\mathrm{CPs}$ composite films. The results indicate not only the synergistic effects of materials with different capacitance

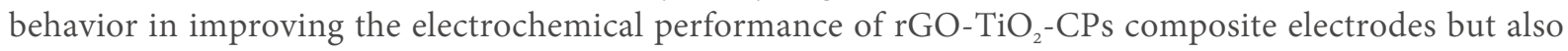
their functional retention after combining with different materials ${ }^{[2,68]}$. These results indicate the synergistic effects EDLC and pseudocapacitance, as well as the universal strategy to prepare flexible and free-standing graphene-based electrodes for WSCs. However, the mechanism property of flexible graphene-based supercapacitors is also an important factor that determines their application in flexible and wearable devices. 
2. Flexible and elastic substrates supported graphene-based electrodes

Although these above flexible and free-standing graphene-based films have already been applied as electrode materials for WSCs, the fabrication of fully flexible electronics with both satisfactory electrochemical performance and mechanical stretchability remains a significant technological hurdle for graphene-based SCs. Thus, some flexible and stretchable substrates such as elastic substrates and textiles provide proper strategies to fabricate flexible and stretchable graphene-based electrodes for WSCs ${ }^{[6,70]}$. ElKady et al. ${ }^{[7]}$ produced rGO films (reduced by laser-scribe) on a polyethylene terephthalate and nitrocellulose membrane (with $0.4 \mathrm{um}$ pore size). The as-prepared films exhibit excellent specific surface area $\left(\sim 1520 \mathrm{~m}^{2} \mathrm{~g}^{-1}\right)$ and conductivity $\left(\sim 1738 \mathrm{~S} \mathrm{~m}^{-1}\right)$, which contributed to the high electrochemical performance when used as electrodes for SCs. In addition to the plastic substrates, new strategies are also developed to directly coat graphene-based SCs on textiles to obtain WSCs. Afroj et al. ${ }^{[72]}$ produced highly conductive, ultra-flexible, and machine-washable graphene-based wearable e-textile electrodes by a simple and scalable pad-dry-cure method [Figure $4 \mathrm{~A}$ and $\mathrm{B}$ ]. The as-prepared graphene-based e-textile has a very low sheet resistance $\left(11.9 \mathrm{~W} \mathrm{sq}^{-1}\right)$ even after 10 home washing cycles. The assembled wearable supercapacitor also possessed good electrochemical performance $\left(2.7 \mathrm{mF} \mathrm{cm}^{-2}\right.$ at $\left.0.1 \mathrm{~mA} \mathrm{~cm}^{-2}\right)$ and stable cycle stability ( $98 \%$ capacitance retained after 15,000 cycles).

Printing technology is also an attractive way to manufacture WSCs being low-cost, time-saving, versatile, and environmentally-friendly ${ }^{[73]}$. During this process, inkjet printing ${ }^{[74]}$, screen printing ${ }^{[75]}$, and $3 \mathrm{D}$ printing $^{[76,77]}$ are usually applied to print the desirable structure on different elastic substrates (papers ${ }^{[78,79]}$, plastic substrates, textiles ${ }^{[80]}$, etc.) to fabricated WSCs. Graphene and graphene-based composite ink was widely used to fabricate WSCs through this method. Figure $4 \mathrm{C}$ displays a printed supercapacitor on flexible glass. Seven printed layers of graphene flakes acted as electrodes and $\mathrm{PVA}-\mathrm{H}_{3} \mathrm{PO}_{4}$ gel acted as electrolyte. The device has small capacitance decay upon bending conditions (from 8.7 to $8.1 \mathrm{mF} \mathrm{cm}^{-2}$ at a curvature radius of $2.75 \mathrm{~cm}$ and a scan rate of $\left.50 \mathrm{mV} \mathrm{s}^{-1}\right)^{[81]}$. To further improve the restack tendency of graphene flakes, Pham et al ${ }^{[82]}$ added a chemical leavening agent in rGO ink to suppress this restacking during the printing process [Figure $4 \mathrm{D}$ and E). Hyun et al. ${ }^{[83]}$ processed a scalable, self-aligned inkjet printing process to manufacture flexible graphene SCs in a high-throughput manner. This strategy offers a promising process and desirable operation metrics including high areal specific capacitance $\left(268 \mu \mathrm{F} \mathrm{cm}{ }^{-2}\right)$, excellent lateral spatial resolution ( $20 \mu \mathrm{m}$ minimum feature size), small footprint $\left(<1 \mathrm{~mm}^{2}\right.$ active device area), and outstanding reliability ( 44 devices with $100 \%$ yield) [Figure $4 \mathrm{~F}$ ]. However, the utilization of electrode materials and the plastic substrates, the breathability, and comfort of the aforementioned studies are not good enough when integrated in wearable devices, especially for skin-touching devices. Therefore, further efforts need to be put into improving these properties. Additionally, strategies from the material point of view including the stretchable, washable, and wear-resistant electroactive materials, stretchable and robust solid-state electrolyte, and proper packaging materials are also extensively needed.

\section{Graphene-based textile fiber electrodes}

Graphene-based fiber electrodes have also been widely applied in WSCs, due to their mechanical flexibility for textiles, light weight, and especially the improved breathability and comfort ${ }^{[8,85]}$. Meng et al. ${ }^{[86]}$ designed a unique all graphene core-sheath fiber, in which a core of graphene fiber is covered with a sheath of threedimensional $(3 \mathrm{D})$ porous graphene network [Figure 5A-C]. High conductivity and greater electrolyte accessible surface area were provided by this hierarchical hybrid structure. The as-prepared flexible allsolid-state fiber supercapacitor was assembled by intertwining two as-prepared graphene fibers with $\mathrm{H}_{2} \mathrm{SO}_{4}$ PVA (polyvinyl alcohol) gel as polyelectrolyte [Figure 5D]. The flexibility is shown in Figure 5F. 
A
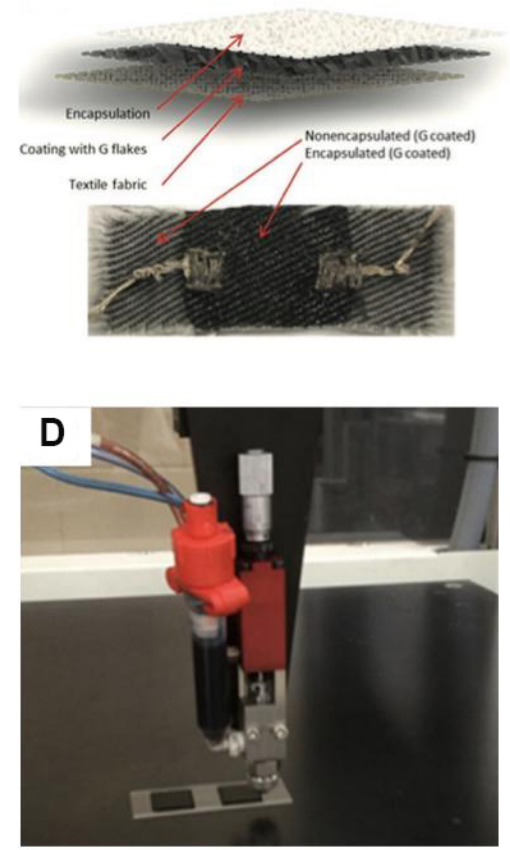

B
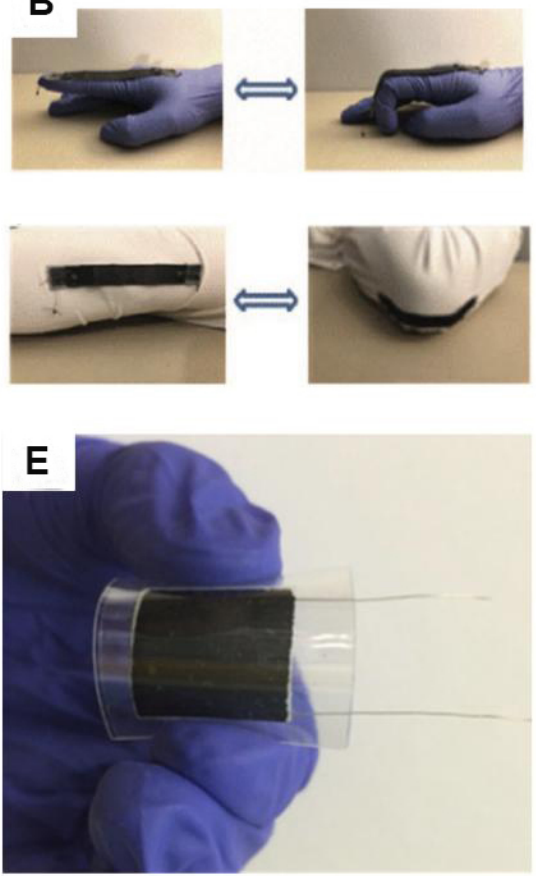

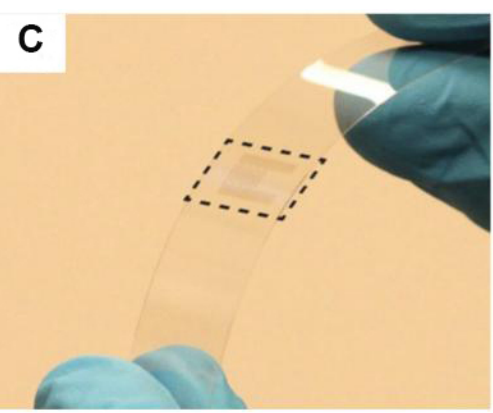

$\mathbf{F}$

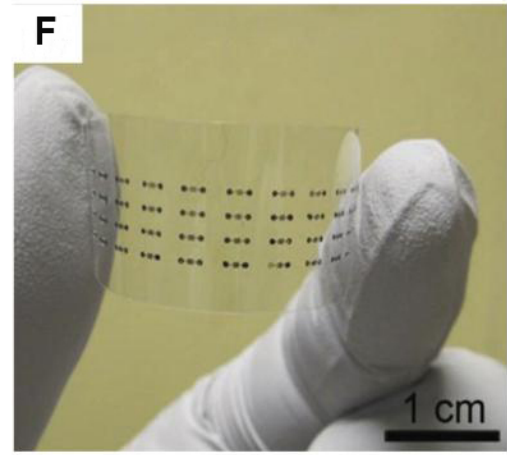

Figure 4. (A) Schematic cross-sectional view of graphene coated, compressed, and encapsulated fabric (top) and a real sample after 10 washing cycles (bottom). (B) Finger joint motion detection, wrist joint detection, and elbow joint motion detection by compressed and encapsulated graphene textile ${ }^{[72]}$. Flexible transparent micro-supercapacitor before electrolyte deposition ${ }^{[81]}$. (B) A snapshot of the GO ink printing on a glass slide. (C) Assembled reduced graphene oxide ( $\mathrm{rGO}$ ) supercapacitor on flexible PET substrate ${ }^{[82]}$. (D) Photograph of a $4 \times 11$ array of graphene MSCs printed on a flexible PET film. (E) Schematic circuit diagram (top) and optical image (bottom) of three devices in a series connection. (F) Galvanostatic charge-discharge (GCD) profiles for the three devices connected in series and parallel, respectively, in comparison with the GCD profiles for a single device. The GCD profiles were acquired at a current of $0.21 \mu \mathrm{A}^{[83]}$.

Additionally, Figure $5 \mathrm{G}$ and $\mathrm{H}$ presents the woven textile and further demonstrates the use of the fiber supercapacitor in electronic textile or clothing integrated devices.

To further improve the capacitance performance of graphene fiber electrodes, specific structures and materials with pseudocapacitance behavior were combined ${ }^{[87,88]}$. Qu et al. ${ }^{[89]}$ produced hollow rGOconductive polymer composite fibers. The high electrical conductivity $\left(4700 \mathrm{~S} \mathrm{~m}^{-1}\right)$ determines their excellent electrochemical performance (fiber shape supercapacitor device with the capacitance and energy density of $304 \mathrm{mF} \mathrm{cm}^{-2}$ and $6.8 \mu \mathrm{W} \mathrm{h} \mathrm{cm}{ }^{-2}$, respectively). Moreover, the as-prepared SC can also be tied into knots, rolled up, and woven into textiles owing to the high flexibility. Although graphene-based fibers have been widely investigated and possess good flexibility and comfort, their stretchability is rarely studied, especially in different temperature conditions. Moreover, the washability of the as-integrated wearable textiles also needs to be further proved, and, to fabricate a proper textile electrode, more textile technologies also need to be developed.

\section{Graphene-based hydrogel electrodes for WSCs}

Graphene-based hydrogels - a kind of porous graphene matrix that can endow electron transfer along the graphene chains, while ions can conduct through the aqueous part within the graphene hydrogels - have attracted lots of attention in the research on WSCs because of their high specific surface area, suitable nano - to micropores and pore network, high conductivity, and multidimensional electron transport 

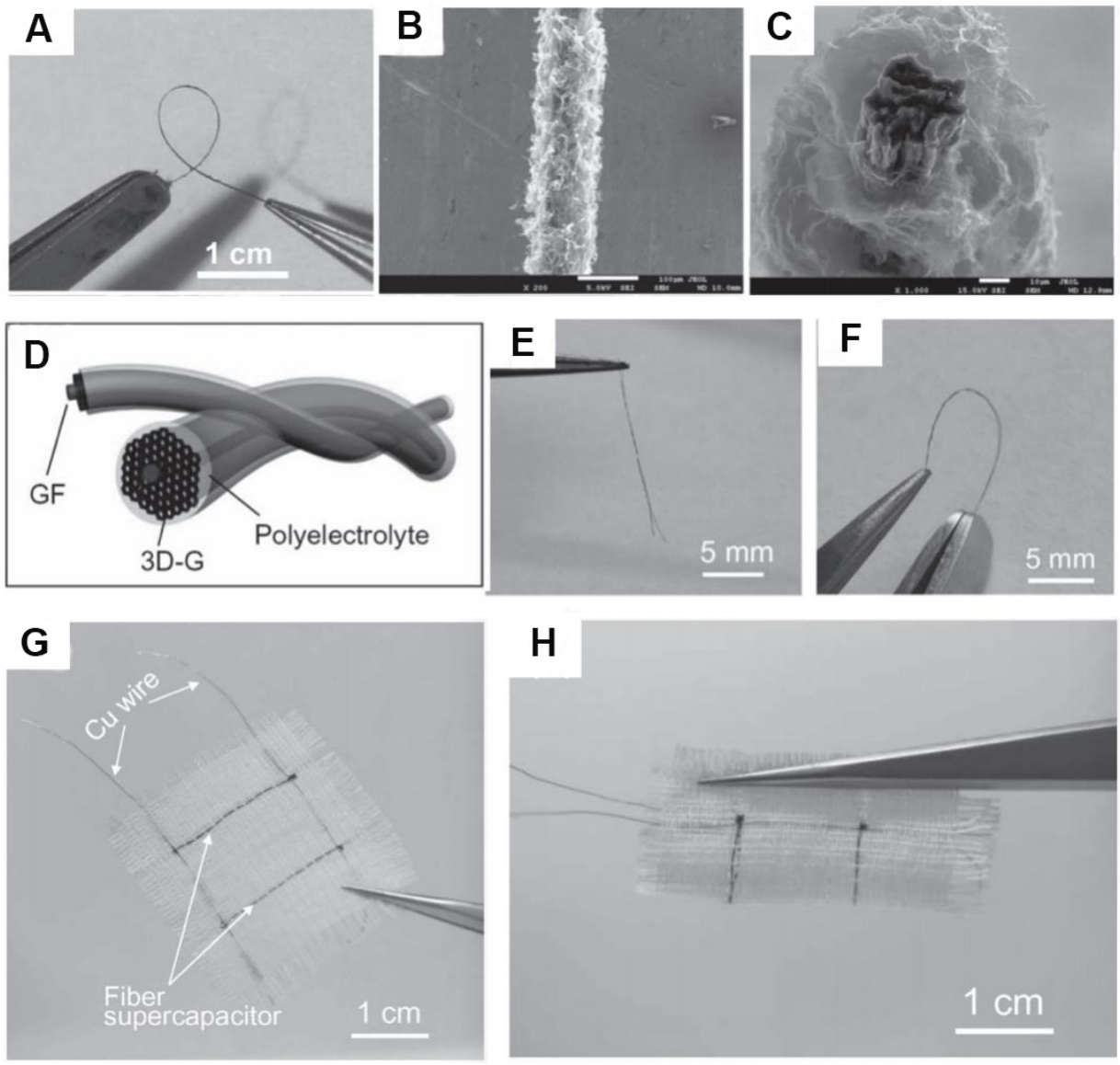

Figure 5. (A) A photograph of a distorted graphene fiber. (B) SEM images of as-prepared graphene fiber. (C) Cross-section view of a graphene fiber showing the core graphene network surrounded by standing graphene sheets. (D) Schematic illustration of a wireshaped supercapacitor fabricated from two twined graphene fibers with a polyelectrolyte. (E, F) Photographs of fiber supercapacitor in free and bending states. $(G, H)$ Photographs of the textile embedded with two graphene fiber supercapacitors in flat and bending states, respectively ${ }^{[86]}$.

pathways ${ }^{[0,91]}$. Figure 6 displays a graphene hydrogel film electrode for WSCs, fabricated by a facile hydrothermal process. The as-prepared graphene hydrogel-based flexible all-solid-state supercapacitor presented good electrochemical performance due to the highly interconnected 3D network structure of graphene hydrogels ${ }^{[5,61]}$. Important advances have also been made to further improve the electrochemical performance and multifunctionality of graphene hydrogel electrodes, including graphene hydrogels composed with $1 \mathrm{D}$ or $2 \mathrm{D}$ metal-compound ${ }^{[92-94]}$ and graphene hydrogels combined with conductive polymers ${ }^{[95-97]}$. Although graphene-based hydrogels have been widely investigated and possess good flexibility and compressivity, their other mechanical properties are still unsatisfactory, especially the poor stretchability, which is caused by the weak $\pi-\pi$ stacking and hydrogen bonding within the reduced graphene oxide matrix that formed during the hydrothermal process.

For decades, although impressive progresses on flexible graphene SCs have already been obtained, the functionality of supercapacitors has always been gained by sacrificing electrochemical performance. Thus, it is still a big challenge to gain flexibility or other functions while still retaining or even improving the capacitance performance of graphene-based electrodes. 

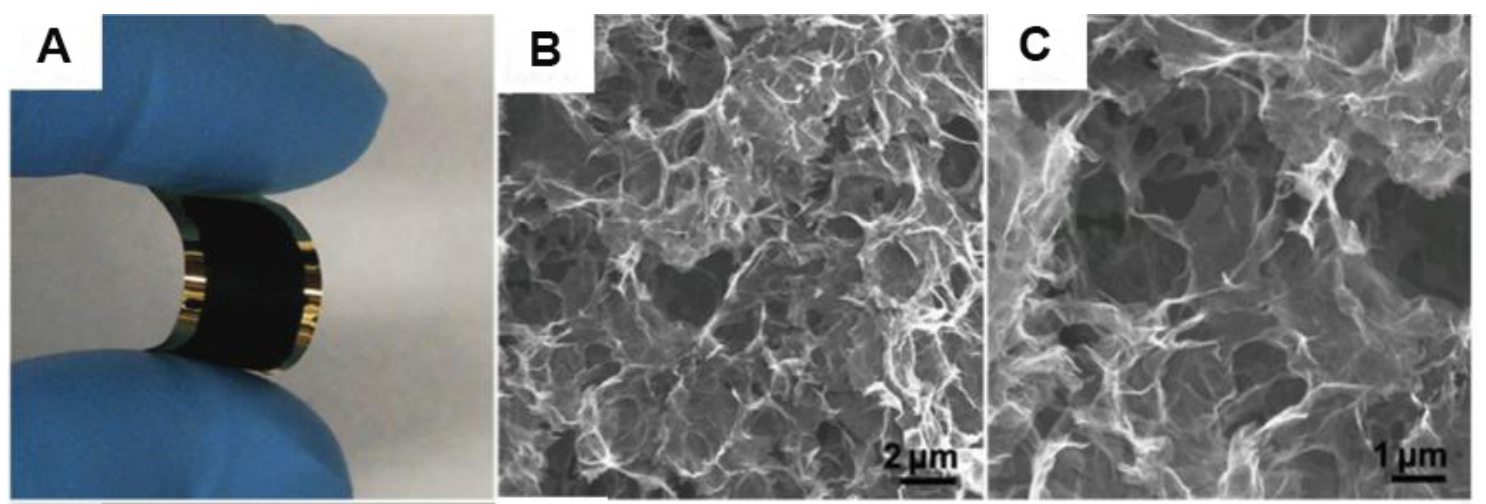

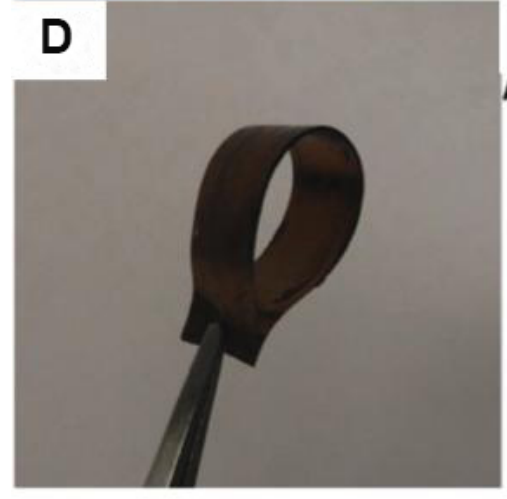

$\mathbf{F}$

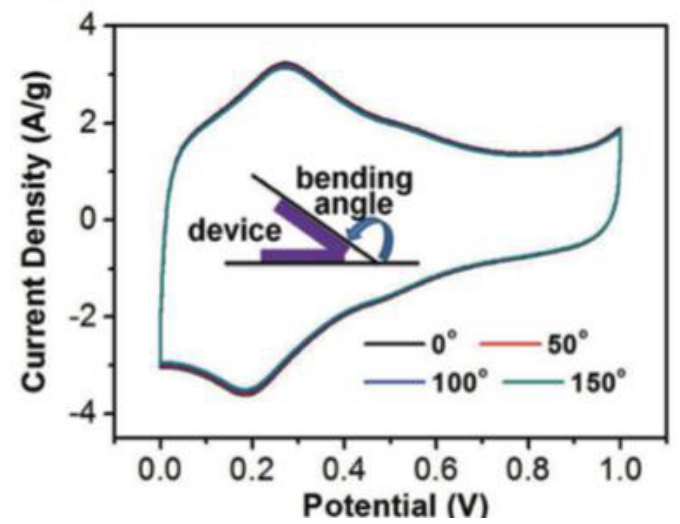

\section{E} Au-coated polyimide

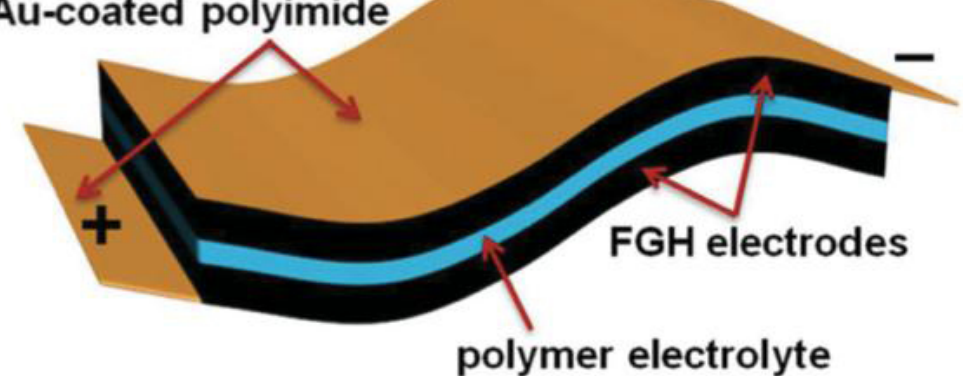

G

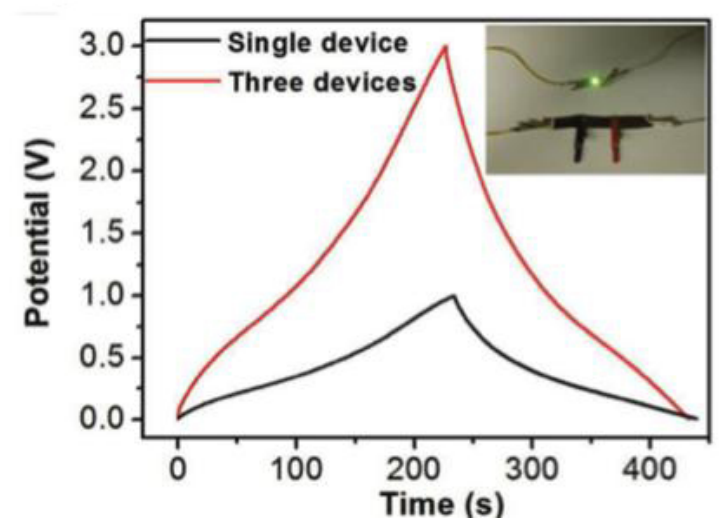

Figure 6. (A) Digital photograph of a flexible FGH thin film electrode. (B) low- and (C) high- magnification SEM images of interior microstructures of the FGH film. (D) Digital photograph of an FGH-based flexible solid-state supercapacitor. (E) A schematic diagram of the solid-state device with $\mathrm{H}_{2} \mathrm{SO}_{4}-\mathrm{PVA}$ polymer gel as the electrolyte and separator. ( $\mathrm{F}$ ) Cyclic voltammetry (CV) curves at $10 \mathrm{mV} \mathrm{s}^{-1}$ of the FGH-based flexible solid-state supercapacitor at different bending angles. (G) One cycle of galvanostatic charge-discharge (GCD) curves at $1 \mathrm{~A} \mathrm{~g} \mathrm{~g}^{-1}$ of a three-supercapacitor in-series group. The inset shows a photograph of a green LED turned on by the tandem device ${ }^{[61]}$.

Carbon nanotube electrodes for WSCs

Carbon nanotubes (CNTs) are another wieldy developed carbon-based electrode material for WSCs and have attracted lots of attention during the preparation of supercapacitors owing to their unique pore and fiber structure, superior electronical properties, and good mechanical and thermal stability ${ }^{[98,99]}$. Tremendous studies have explored CNT-based fiber SCs corresponding to the fibrous structure nature of CNTs ${ }^{[100,101]}$. Other electroactive materials have also been exploited to combine with CNTs to fabricate fibrous structures for textile SCs and improve the electrochemical performance ${ }^{[102-104]}$. 
Similar to graphene, combining CNTs with elastomer substrates is the most common strategy to fabricate flexible or stretchable electrodes for WSCs by transferring ${ }^{[105,106]}$ or coating ${ }^{[107-109]}$ methods. Recently, seamlessly connected graphene/carbon nanotube hybrids with covalent interconnections were developed by growing vertically aligned CNTs on graphene layers. This special 3D structure could efficiently prevent the aggregation and possess large surface area and excellent electrical properties. A free-standing tubular structure of seamless graphene/CNTs textile structure was obtained by etching off the original nickel textile. After being deposited with polyaniline (PANi), this as-prepared electrode material exhibited a high area specific capacitance of $164 \mathrm{mF} \mathrm{cm}^{-2}$. Due to the unique textile-like structure, the assembled supercapacitor exhibited not only excellent flexibility but also high stretchability of $200 \%^{[110]}$.

Printing technology is also a popular way to fabricate CNT-based electrodes for WSCs. Lee et al. ${ }^{[80]}$ assembled printed SCs composed of multiwalled carbon nanotube electrodes and ionic liquid/thiolene polymer network skeleton $/ \mathrm{SiO}_{2}$ nanoparticle-based gel electrolytes on a T-shirt by printing-assisted aesthetic clothing designs [Figure 7]. This simple and scalable printing process, combined with the properly designed electrodes and electrolytes, make the printed WSCs possess excellent form factors, mechanical flexibility, and thermal stability. Furthermore, the printed T-shirts maintain their electrochemical activity even upon exposure to laundering, wringing, ironing, and folding, which are common activities for clothing, demonstrating their potential in wearable electronics. This study provided a useful strategy to produce washable and comfortable WSCs, however, the wearable demand in the everyday-use level still has not been investigated and efforts still need to be done to realize the commercial WSCs.

\section{Polymer-based electrodes for WSCs}

Conductive polymer-based electrodes for WSCs

Conductive polymers, such as polypyrrole (PPy), PANi, polythiophene (PTh), and poly (3,4ethylenedioxythiophene) (PEDOT), are another kind of electrode materials that have been widely applied in supercapacitors owing to their pseudocapacitance behaviors ${ }^{[11-113]}$. As shown in Figure 3, the Young's modulus, deformability, and conductivity of conductive polymers is more suitable than those of carbon- or metal oxide-based materials for WSCs. Especially the conductivity can reach up to $10^{4} \mathrm{~S} \mathrm{~cm}^{-1}$ by different doping levels or dopants ${ }^{[112]}$.

However, the poor stability of conductive polymers, which comes from their backbone shrink and deterioration during the electrochemical process, will decline the power density and energy density of the whole devices and hinder their further applications ${ }^{[1,21,12]}$. Thus, fabricating conductive polymers on a designed surface structure or combined with other electroactive materials such as carbon-based ones or metal compounds are the most popular strategies to utilize the advantages of each material and achieve high electrochemical performances. For WSCs, the above-mentioned method is applicable and achievable by using elastic substates. Vertical PPy nanotube arrays and carbon nano-onions grown on spandex fabric to fabricate a flexible and stretchable electrode for WSCs are shown in Figure 8A. The assembled stretchable supercapacitors exhibit $64 \mathrm{~F} \mathrm{~g}^{-1}$, and they also present capacitance retention of $99 \%$ at a strain of $50 \%$ after 500 cycles. This as-prepared stretchable supercapacitor device can provide a stable energy supply under different bending conditions for practical applications [Figure $8 \mathrm{E}-\mathrm{G}]^{[114]}$.

Printing technology is also applied in fabricating conductive polymer-based electrodes for WSCs. Recently, a low-cost, easy-to-fabricate, and air-stable PANi ink was developed through a facile assemble-disperse strategy. The printable SC derived from as-prepared CP ink, as shown in Figure 9A, delivers high areal capacitance of $96.6 \mathrm{mF} \mathrm{cm}^{-2}$, large volumetric capacitance of $26.0 \mathrm{~F} \mathrm{~cm}^{-3}$, and considerable energy density of $2.4 \mathrm{mWh} \mathrm{cm}^{-3}$ at $238.3 \mathrm{~mW} \mathrm{~cm}^{-3}$ [Figure 9] ${ }^{[115]}$. 

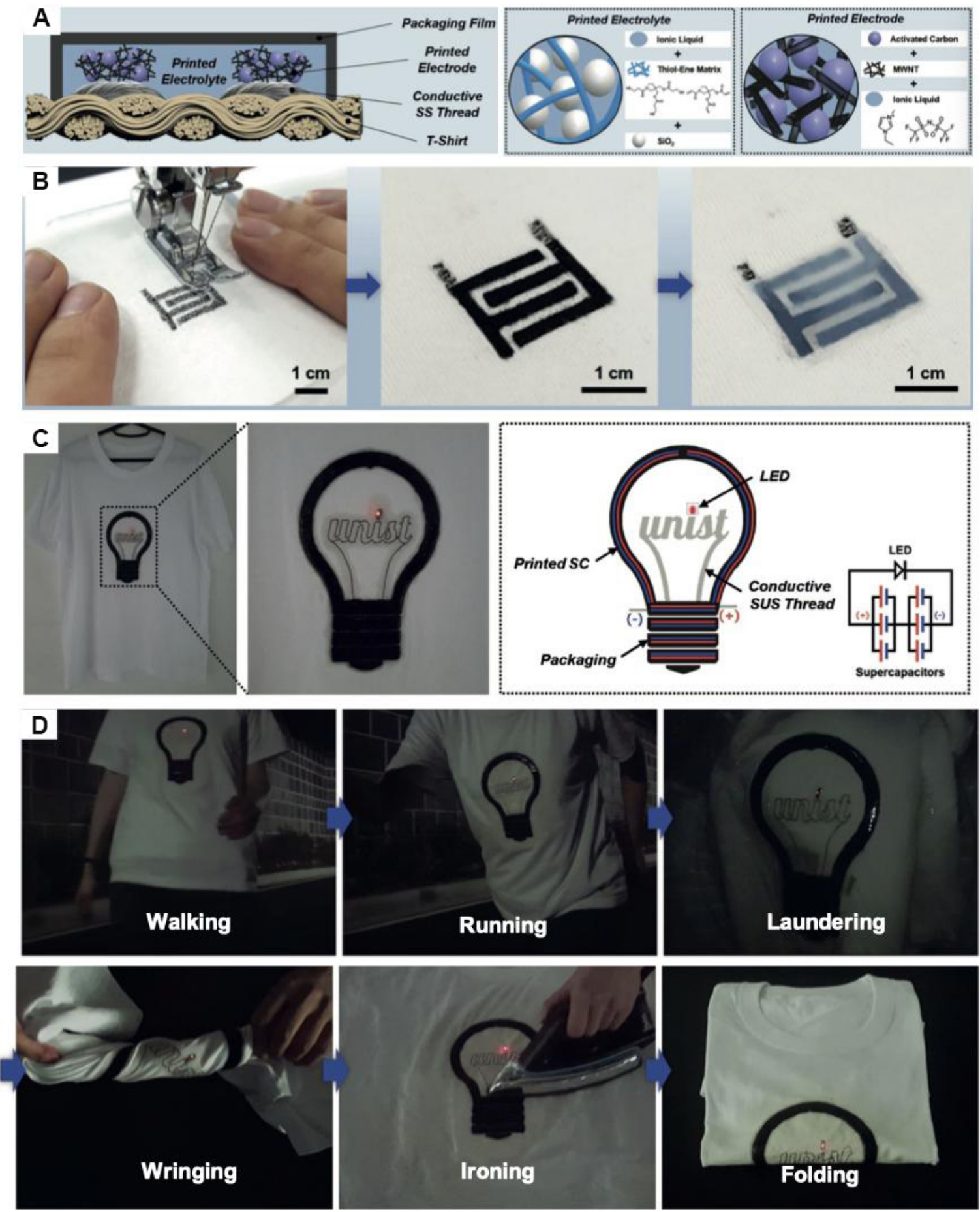

Figure 7. (A) Schematic representation illustrating an SC-printed T-shirt and its major components [SS thread current collector, printed electrode (AC/MWCNT/ionic liquid), printed gel electrolyte (ionic liquid/thiolene polymer network skeleton/SiO ${ }_{2}$ nanoparticles), and packaging film]. (B) Schematic representation depicting the printing-based, stepwise fabrication procedure of the SC-printed T-shirt. Application of a SC-printed T-shirt as a potential electronic garment. (C) Photographs and a conceptual illustration of the bulb-shaped $\mathrm{SC}\left(3.0 \mathrm{~V} / 15 \mathrm{mF} \mathrm{cm}^{-2}\right.$, scan rate $\left.=2.0 \mathrm{mV} \mathrm{s}^{-1}\right)$ that powered an LED lamp. The SC was directly printed on a white T-shirt and connected to the LED lamp using conductive SS thread. (D) Photographs of the SC-printed T-shirt upon exposure to various wearable test modes (walking, running, laundering, wringing, ironing, and folding) encountered in the daily wear of garments ${ }^{[80]}$. 

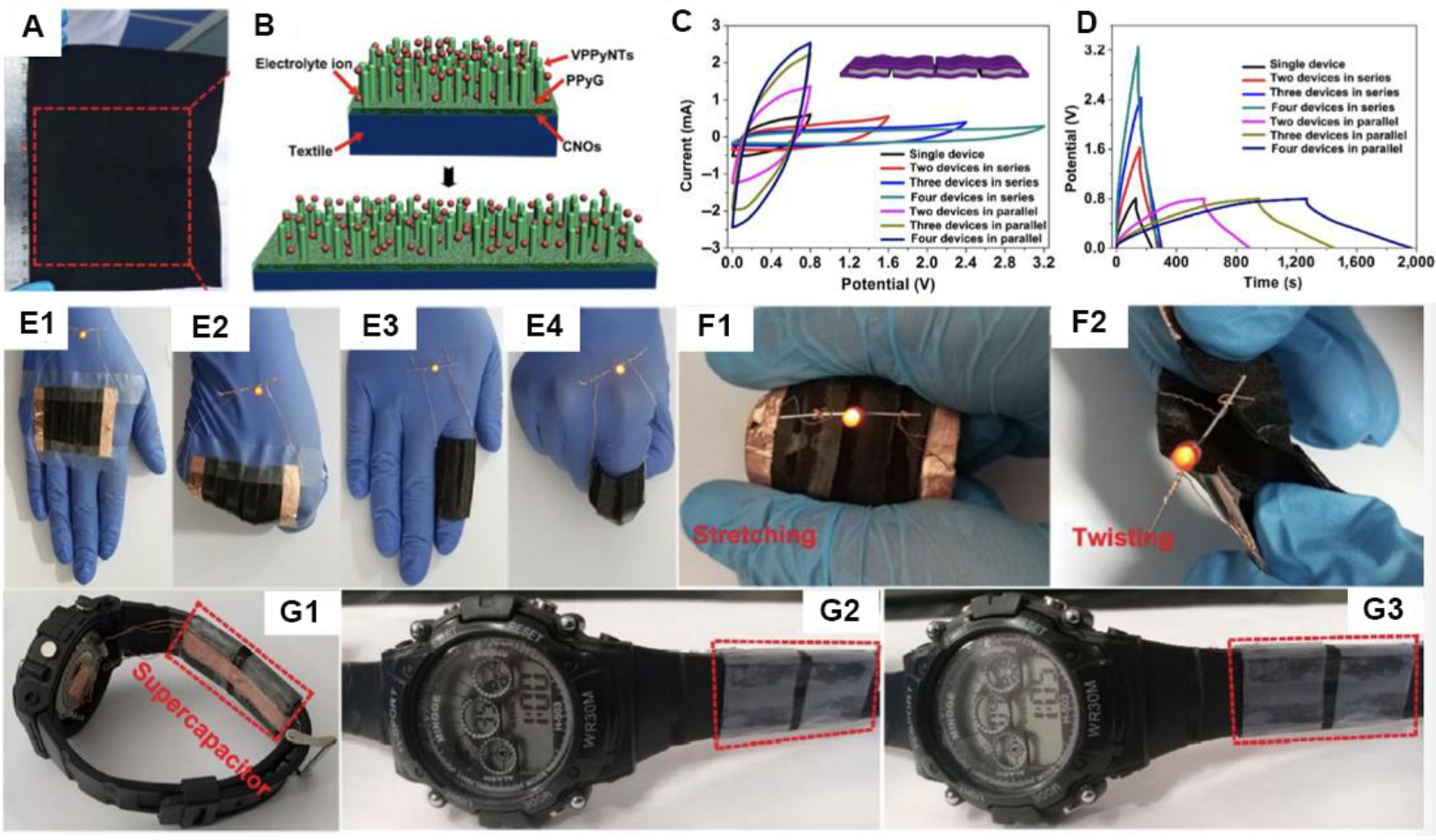

Figure 8. (A) Photograph of a large-area piece of spandex fabric after the growth of the PPy structure. (B) Schematic illustration of the conductivity retaining mechanism of the stretchable WSC based on a textile electrode during stretching. (C) CV and (D) GCD curves of the stretchable SC composed of multiple devices in series and parallel under scan rate of $10 \mathrm{mV} \mathrm{s}^{-1}$ and current density of $0.2 \mathrm{~A} \mathrm{~g}^{-1}$. The inset is the cartoon of a stretchable SC device composed by four single devices in series. (E1-E4) Photographs of a red LED lit up by the stretchable SC as a wearable power supply attached on the human hand and finger under the original and stretchable states. (F1, F2) Photographs of a red LED bubble lit up by the as-prepared SC under stretch and twist condition. (G) Photographs of an electronic watch powered by a stretchable supercapacitor ${ }^{[114]}$.
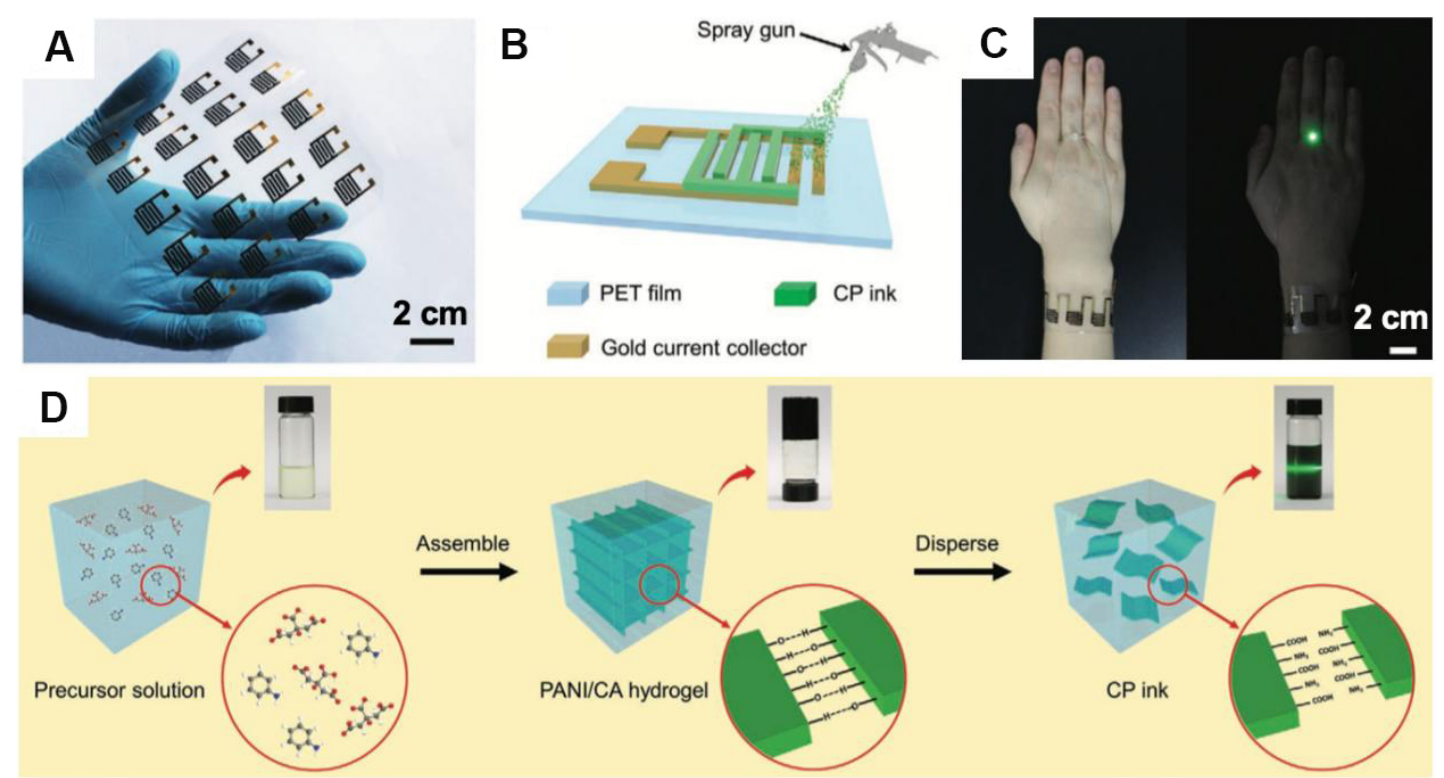

Figure 9. (A) Flexible WSC array derived from air stable conductive polymer ink. (B) Schematic illustration for the fabrication of printed WSCs with a mask-assisted spray-coating method. (C) Integrated circuit of a WSC wristband that can successfully power a green LED bulb. (D) Air-stable conductive CP ink can be easily prepared through a facile assemble-disperse strategy, in which PANi and citric acid assemble into hydrogel in the assemble stage and sequentially disperse well in water to form CP ink in the disperse stage ${ }^{[115]}$. PET: Polyethylene terephthalate; CP: conductive polymer. 
Although the deposition of conductive polymers on stretchable elastic substrates, such as polyurethane, polydimethylsiloxane, and thermoplastic copolyester, is the most common strategy to obtain flexible and stretchable electrodes for WSCs, these conventional stretchable electrodes are limited by the deformability of the substrate during the stretching or deformation process ${ }^{[116,117]}$. Recently, Yang et al..$^{[18]}$ developed an additive-free, free-standing stretchable electrodes by a $3 \mathrm{D}$ printing process based on poly $(3,4$ ethylenedioxythiophene):polystyrene sulfonate (PEDOT:PSS) ink. Figure 10A displays the schematic illustration of the fabrication process of the conducting polymer electrode. Different 3D patterns were firstly prepared on a glass substrate; after being pulled off, different free-standing stretchable electrodes were obtained [Figure 10B]. These well-designed arc-shaped microstructures provided a uniform stress area and reduced the peak strain in the electrode, which led to excellent flexibility and extreme stretchability (maximum elongation of 150\%; Figure 10C). The quasi-solid-state symmetric supercapacitor, further assembled by hybrid polymer/CNT electrode, exhibited a high energy density of $0.065 \mathrm{~mW} \mathrm{~h} \mathrm{~cm}^{-2}$ and maintained excellent capacitance after 14,000 cycles. However, the mass loading of the ink during the printing process still hinders their development. Furthermore, the comfort also needs to be further considered.

\section{Conductive polymer-based hydrogels for WSCs}

The intrinsic soft/wet properties of hydrogels provide an excellent interface between the electronic transporting phase (electrode) and the ionic transporting phase (electrolyte), between biological and synthetic systems, and between soft and hard materials ${ }^{[29,88]}$. Moreover, according to the mechanical properties of gels [Figure 3], hydrogels are very promising electrode materials for WSCs. Therefore, hydrogel electrodes are recently widely explored. However, most hydrogels with unconducive polymer backbones cannot fulfill the demands of SCs. To resolve this issue, the most common strategy is using conductive polymers, e.g., PTh, PEDOT, PANi, and PPy, as backbones to fabricate hydrogels with unique structural and electronic properties (conductivity and pseudocapacitance behavior) ${ }^{[119-122]}$. PANi matrix hydrogels [Figure 11A and B] were first reported by using phytic acid as the gelator dopant. These asprepared PANi hydrogels show excellent conductivity of about $0.11 \mathrm{~S} \mathrm{~cm}^{-1}$ and high capacitance as electrode for supercapacitors (480 $\mathrm{F} \mathrm{g}^{-1}$ and $83 \%$ capacitance retention after 10,000 cycles) ${ }^{[123,124]}$. Similar studies using acid-linked conductive polymer-based hybrid hydrogels can also combine functional particles to form functional hydrogel electrodes for WSCs, For example, Ag nanoparticles were introduced into a folic acid cross-linked PANi hydrogel to improve the conductivity and energy storage property of the as-prepared hydrogels ${ }^{[125]}$. To further improve the electrical property of conductive polymer based hydrogels, multivalent metal ions $\left(\mathrm{Fe}^{3+}, \mathrm{Mg}^{2+}\right.$, and $\left.\mathrm{Cu}^{2+}\right)$ were also used to cross-link the conductive polymer chains as the gelator dopant to form conductive polymer-based electrode hydrogels free of insulating components ${ }^{[122,126,127]}$.

In conductive polymer-based hydrogels, the conjugated structures of polymers are inherently rigid and fragile, which impairs the mechanical properties that are also significant factors for WSCs. New double network and low temperature strategies were applied to prepare great stretchable conductive polymer-based hydrogels with a hierarchical micro-/nanostructure ${ }^{[128]}$. The as-prepared hydrogels possess good toughness (29-fold enhancement) and electrochemical performance (specific and areal capacitance is $888 \mathrm{~F} \mathrm{~g}^{-1}$ and $2097 \mathrm{mF} \mathrm{cm}{ }^{2}$, respectively) while slightly sacrificing the conductivity $\left(5.99 \mathrm{mS} \mathrm{cm}^{-1}\right.$; after adding phytic acid, the conductivity is $91 \mathrm{mS} \mathrm{cm}{ }^{-1}$ ). However, it is still a challenge for researchers to prepare conductive polymer-based hydrogels with high stretchability and high conductivity simultaneously. Other functions, such as biocompatibility, can be realized by introducing biomolecules into conductive polymer-based hydrogels ${ }^{[129]}$. Few works have applied such hydrogel electrodes in biocompatible or implantable supercapacitors. Hence, it is also important to further modify the conductive polymer hydrogels or combine other electroactive materials to endow more unconventional functions or specific properties for conductive 


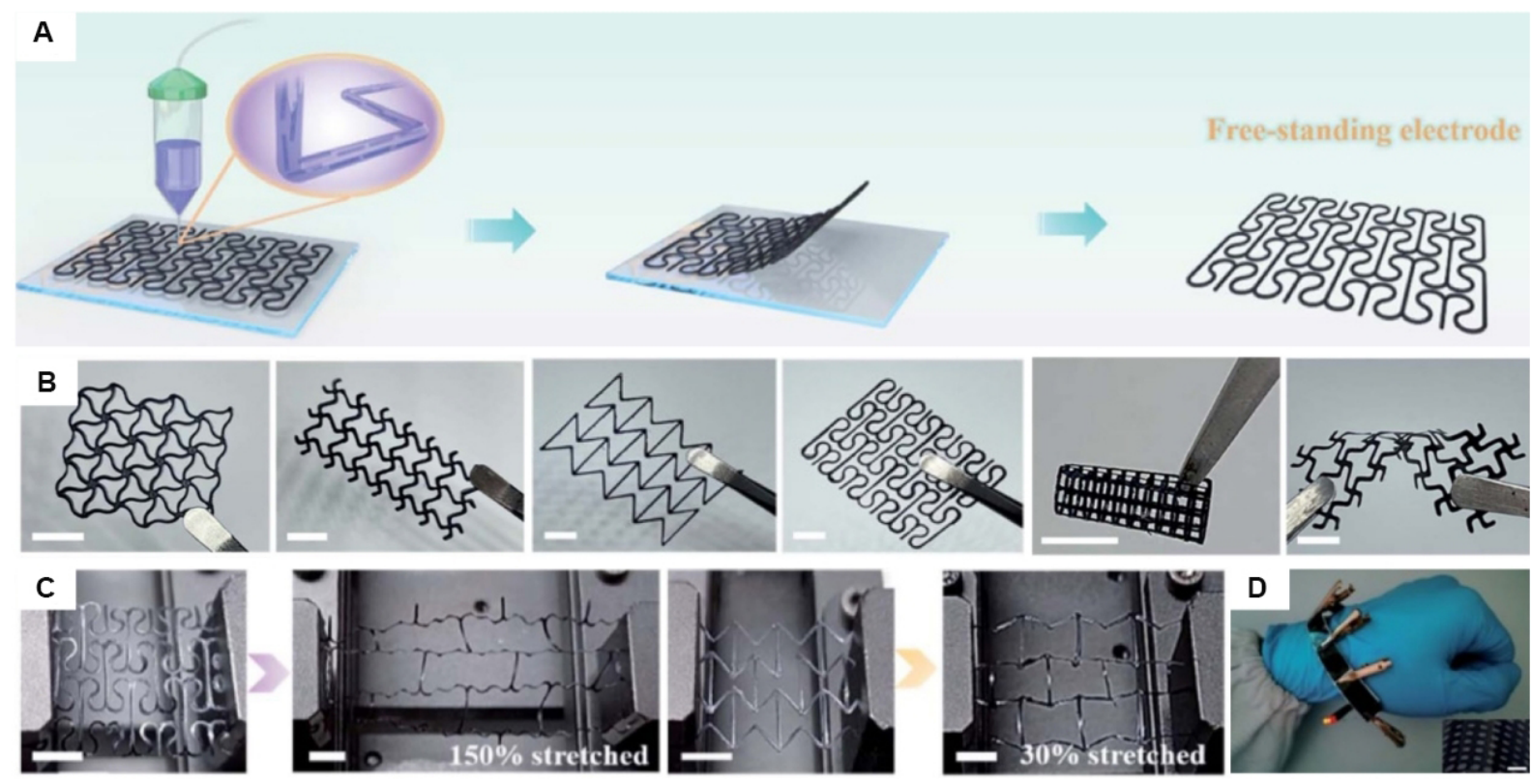

Figure 10. (A) Schematic illustration of the fabrication process of the conducting polymer electrode. (B) Images of as-printed electrodes with di erent structures. Scale bars: $5 \mathrm{~mm}$. (C) S-hinged and re-entrant structure electrodes in the maximum stretch state. Scale bars: 6 $\mathrm{mm}$. (D) Photographs of the wearable PEDOT:PSS/CNT quasi-solid-state symmetric supercapacitor lighting up a red LED bubble. The inset shows magnified optical image of the electrode. The scale bar is $1 \mathrm{~mm}^{[118]}$.

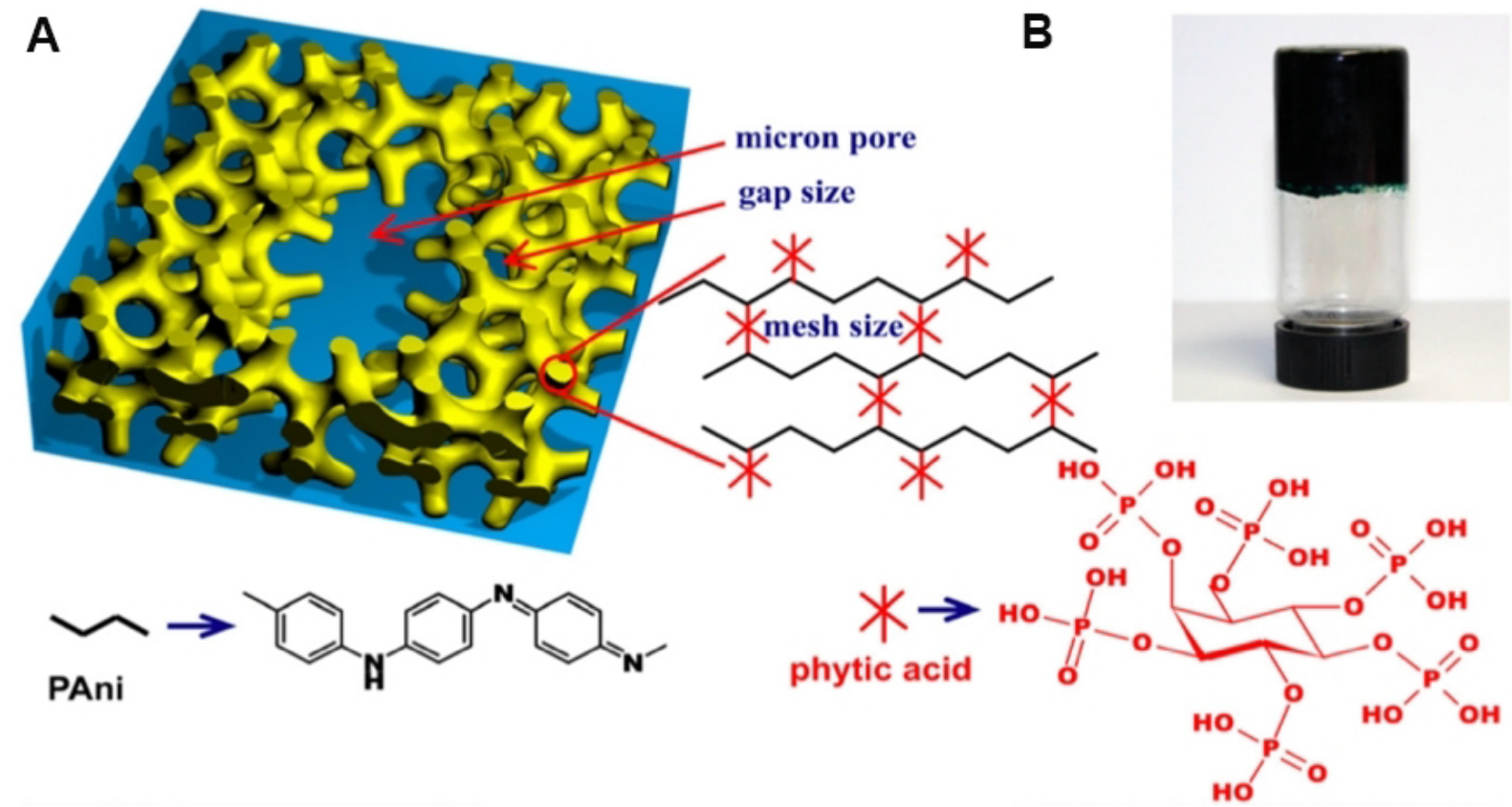

Figure 11. (A) Schematic illustrations of the 3D hierarchical microstructure of the gelated PANi hydrogel where phytic acid plays a role as a dopant and a crosslinker. The three-level hierarchical porosity of angstrom, nanometer, and micron size pores is highlighted by red arrows. (B) A photograph of the PANi hydrogel inside a glass vial ${ }^{[123]}$. PANi: Polyaniline.

polymer-based hydrogel electrodes for WSCs. 


\section{Metal-containing electrodes for WSCs}

Metal compound electrodes for WSCs

Tremendous efforts have been made in the investigation of metal compound electrode materials for SCs, including meta oxides, metal nitrides, metal sulfides, and metal carbides ${ }^{[26]}$. According to the energy storage mechanism of supercapacitors, metal-based electrodes which possess pseudocapacitance behavior could obtain higher electrochemical performance compared with carbon-based electrodes, which present EDLC behavior. However, the deformability and Young's modulus of bulk and rigid metal compounds cannot meet the demands of electrodes for WSCs. Down scaling is the most common strategy to improve the properties of metal compounds for WSCs. Nano/microstructure metal compounds are the most popular candidate electrodes, but they have to face the decline of conductivity. Thus, metal compounds composed with carbon-based or conductive polymers are the most popular strategy, which not only improves the conductivity of the whole electrode for WSCs but also enhances the electrochemical performance by the synergistic effect of both pseudocapacitance behavior and EDLC behavior. In our previous work, metal oxides (nano- $\mathrm{TiO}_{2}$ and $\mathrm{Co}_{3} \mathrm{O}_{4}$ ) composed with graphene and conductive polymers (PPy, PANi, and PTh) were applied as free-standing, flexible electrodes for WSCs [Figure $12 \mathrm{~A}-\mathrm{C}]^{[2,130,131]}$. To further improve the conductivity and electrochemical properties of the metal oxides, series of metal compound electrodes consisting of metal oxides, bimetal oxides, and metal nitrides $\left(\mathrm{NiCoO}_{2} @ \mathrm{Co}_{3} \mathrm{O}_{4} @ \mathrm{Co}_{2} \mathrm{~N}, \mathrm{NiMoO}_{4} @ \mathrm{MoO}_{3}\right.$ $@ \mathrm{Mo}_{2} \mathrm{~N}$, and $\mathrm{NiFe}_{2} \mathrm{O}_{4} @ \mathrm{Fe}_{3} \mathrm{O}_{4} @ \mathrm{Fe}_{2} \mathrm{~N}$ ) were also combined with rGO to produce electrodes for flexible allsolid-state supercapacitors [Figure 12D-F]. These studies not only proved the synergistic effects of different capacitance behaviors but also proved a universal designed strategy to produce hybrid materials for highperformance functional WSCs ${ }^{[17,132]}$.

In addition to the free-standing, flexible, and paper-like metal compound-containing hybrid electrodes for WSCs, fiber-shaped hybrid electrodes have been developed ${ }^{[133,134]}$. Salman et al. ${ }^{[135]}$ integrated tungsten with rGO to fabricate hybrid fiber supercapacitors with high electrochemical performance $\left(16.29 \mathrm{~F} \mathrm{~cm}^{-3}\right.$ at $0.05 \mathrm{~A}$ $\mathrm{cm}^{-3}$, energy density of $1.448 \mathrm{~mW} \mathrm{~h} \mathrm{~cm}^{-3}$, and $84.7 \%$ capacitance retention after 10,000 cycles). To further improve the stretchability of metal compound-based electrodes, printing technology and elastic substrates have also been applied ${ }^{[136,137]}$. Recently, an implantable and biodegradable printed micro-supercapacitor was prepared by a facile super-assembly manufacturing and screen-printing strategy. The produced and optimized implantable and biodegradable supercapacitor which was super-assembled by a patterned Zn@PPy electrode and NaCl/agarose electrolyte exhibits energy density of $0.394 \mathrm{~mW} \mathrm{~h} \mathrm{~cm}^{-2}$ and can be fully degraded in vivo in 30 days without any adverse effects in the host organism ${ }^{[46]}$. This work gives us inspiration to study transient electronics for further implantable electronics. However, the design and choice of electrodes and electrolytes of implantable SCs is still the most challenging part. The materials must possess high biocompatibility, be soft (compatible with soft organs and tissues), and have no toxicity. For electrolytes, $\mathrm{NaCl}$ or phosphate-buffered saline could be proper since they already exist in the human body. Additionally, to further adapt to the unique physiological environment of the human body, implantable SCs with the ability to be stretched, compressed, bent, twisted, and deformed into arbitrary shapes must be further studied and developed.

\section{Metal-organic frameworks based electrodes for WSCs}

Metal-organic frameworks (MOFs) have attracted significant interests since the studies conducted by Hoskins and Robson ${ }^{[138,139]}$. These structures are defined by their assembly from metal/cluster-ligand coordination with multimodal ligands to fabricate extended, different dimensional porous networks with characteristics of high surface area, permeability to foreign entities, and structural tailorability ${ }^{[140-143]}$. MOFs as an important family of metal-containing materials have also been well studied as electroactive materials for SCs due to their porous structure and active sites, which could contribute both EDLC behavior and pseudocapacitance behavior. 
A

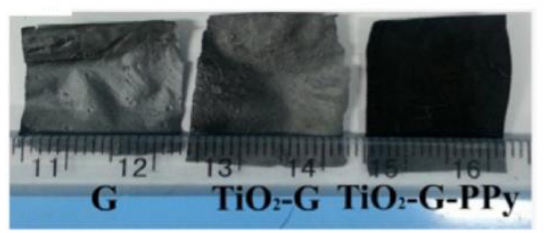

D

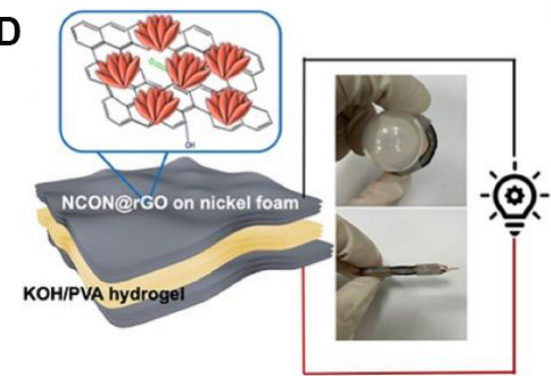

B
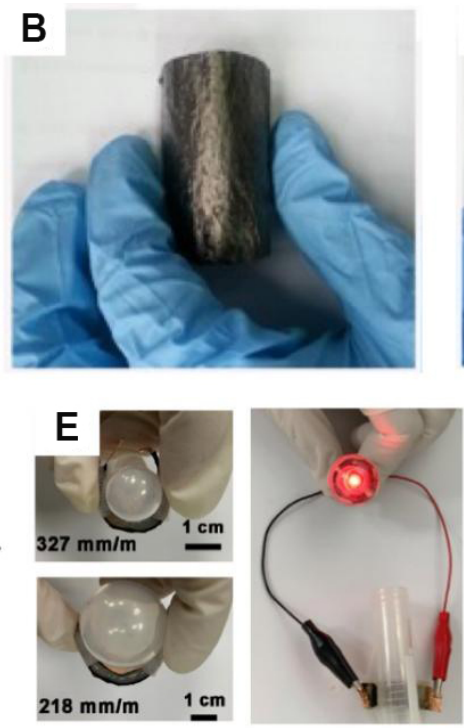
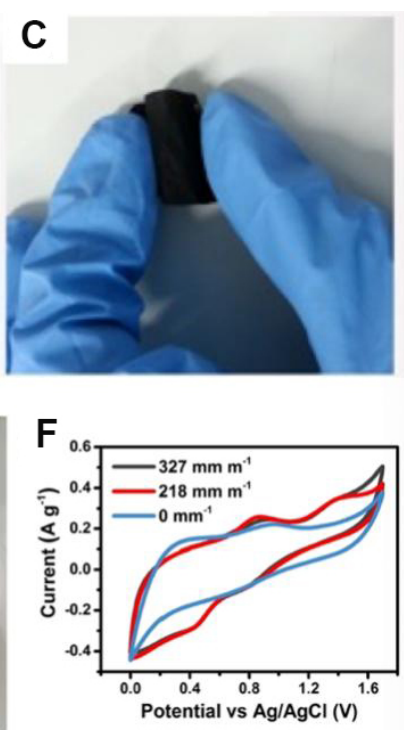

Figure 12. (A) Photographs of $\mathrm{rGO}$ and $\mathrm{rGO}$-based composite films including free-standing and flexible rGO films (denoted as $\mathrm{G}$ ), $\mathrm{TiO}_{2}$ rGO film, and $\mathrm{TiO}_{2}-\mathrm{rGO}-\mathrm{PPy}$ film. (B) Bent flexible $\mathrm{TiO}_{2}-\mathrm{rGO}$ film. (C) Bent flexible $\mathrm{TiO}_{2}-\mathrm{rGO}-\mathrm{PPy}$ film ${ }^{[130]}$. (D) Schematic illustration of the as-assembled hybrid high-performance, flexible, and all-solid-state supercapacitor. (E) CV curves of FSS at different curvatures. (F) Photographs of FSS under different bending conditions and an LED light bulb lit up by FSS at the curvature of $\delta=327 \mathrm{~mm} \mathrm{~m}^{-1[17]}$. rGO: Reduced graphene oxide; PPy: polypyrrole.

The utilization of MOFs include two aspects: On the one hand, MOFs are utilized as novel templates for preparing porous metal compounds or carbons. They can be applied as electrode materials for WSCs engaging with wearable substrates due to the nano/microscale nature of MOFs. Zhou et al. ${ }^{[144]}$ directly grew MIL-88-Fe MOF-derived spindle-like $\mathrm{Fe}_{2} \mathrm{O}_{3} @ \mathrm{C}$ on oxidized CNT fibers to produced fiber-shaped electrodes for WSCs. The assembled wearable asymmetric supercapacitor possessed high specific capacitance of 201.4 $\mathrm{mF} \mathrm{cm}^{-2}$, energy density of $135.3 \mu \mathrm{Wh} \mathrm{cm}^{-2}$, and good stability (97.1\% retains after 4000 cycles). Zhou et al.$^{[145]}$ prepared PPy-coated conductive bacterial cellulose (BC) membranes via a ZIF- 67 sacrificing polymerization process. This flexible and binder-free electrode exhibits high electrochemical performance due to the recovered electrolyte accessible channels afforded by the well-ordered PPy alignments along the BC nanofibers [Figure 13A]. However, in these studies, most MOFs only serve as the sacrificial templates/precursors with facile structural collapse and loss of intrinsically large surface area and pore volume.

On the other hand, MOFs with pseudocapacitive redox centers can be directly used as electrode materials $s^{[146-149]}$. Although the specific structure of MOFs can be kept, the poor intrinsic conductivity of pristine MOFs is also a challenging issue that needs to be resolved. To solve this issue, an extensively studied strategy is combining with other electronically conducting materials including conductive polymers and carbon-based materials which possess both good electronic conductivity and excellent redox or EDLC characteristics $^{[150-152]}$. Another effective strategy is exploring novel MOFs with intrinsic conductivity ${ }^{[153]}$. Figure $13 \mathrm{~B}$ and $\mathrm{C}$ shows intrinsically conductive $\mathrm{Cu}$-MOF nanowire arrays on a free-standing PPy membrane electrode. The conductive $\mathrm{Cu}-\mathrm{MOF}$ nanowire arrays endowed the electrode with high conductivity and high surface area for the accessibility of electrolyte, whereas the PPy membrane provided flexibility, efficient charge transfer skeleton, and extra capacitance. The assembled flexible supercapacitors possessed exceptional electrochemical performance (areal capacitance of $252.1 \mathrm{mF} \mathrm{cm}^{-2}$, energy density of $22.4 \mu \mathrm{Wh} \mathrm{cm}{ }^{-2}$, power density of $1.1 \mathrm{~mW} \mathrm{~cm}^{-2}$, and $90 \%$ capacitance retention after $\left.8000 \mathrm{cycles}\right)^{[154]}$. Additionally, to further improve the stretchability of MOF-based electrodes for WSCs, printing strategies 

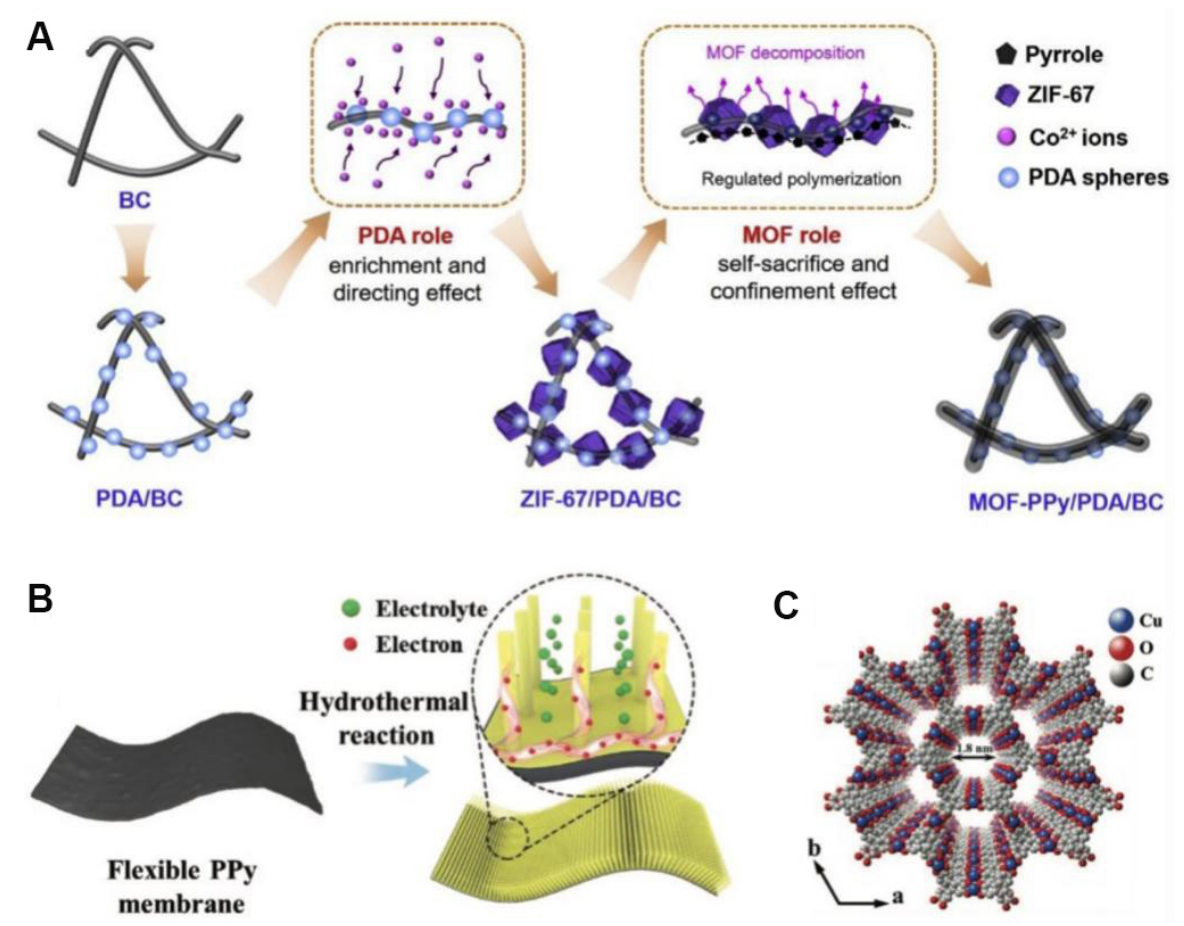

Figure 13. (A) Illustration of PPy-coated conductive bacterial cellulose (BC) membranes via a ZIF-67 sacrificing polymerization process $^{[145]}$. (B) Illustration of the preparation of Cu-MOF nanowire arrays on free-standing PPy membrane electrode. (C) Simulated crystal structure of $\mathrm{Cu}-\mathrm{MOF}^{[154]}$. PPy: Polypyrrole.

have also been applied to print MOF hybrids on elastic substrates ${ }^{[152,155]}$.

MXene-based electrodes for WSCs

Since the first discovery of MXene in 2011 ${ }^{[156]}$ (a family of 2D transition metal carbides or nitrides), it has been intensively investigated, resulting in the discovery of more than 30 compositions including $\mathrm{Ti}_{2} \mathrm{CT}_{x}, \mathrm{Nb}_{2}$ $\mathrm{CT}_{x}, \mathrm{~V}_{2} \mathrm{CT}_{\mathrm{x}}, \mathrm{Ti}_{3} \mathrm{C}_{2} \mathrm{~T}_{\mathrm{x}}, \mathrm{Mo}_{2} \mathrm{TiC}_{2} \mathrm{~T}_{\mathrm{x}}, \mathrm{Mo}_{2} \mathrm{Ti}_{2} \mathrm{C}_{3}, \mathrm{Ti}_{\mathrm{y}} \mathrm{Nb}_{2-\mathrm{y}} \mathrm{CT}_{\mathrm{x}}$, and $\mathrm{Nb}_{\mathrm{y}} \mathrm{V}_{2-\mathrm{y}} \mathrm{CT}_{\mathrm{x}}$, along with dozens more explored by computational methods ${ }^{[157,158]}$. The general formula of MXene is $\mathrm{M}_{n+1} \mathrm{X}_{\mathrm{n}} \mathrm{T}_{\mathrm{x}}(n=1-4)$, where $\mathrm{M}$ represents transition metals, $\mathrm{A}$ is an element of Groups 13-15 in the periodic table, $\mathrm{X}$ is carbon or nitrogen, and $\mathrm{T}_{\mathrm{x}}$ is surface functional groups $(\mathrm{OH}, \mathrm{O}, \mathrm{Cl} \text {, and } \mathrm{F})^{[159,160]}$. Owing to the above unique structure and components, MXenes possess specific physical and chemical merits such as great miscibility, high surface area to volume ratio, accessible active sites, surface charge state and electron-rich density, and absorption of electromagnetic waves, indicating their potential in electrochemical energy storage and conversion, electromagnetic shielding, wearable sensors, and personal thermotherapy ${ }^{[16,162]}$. Moreover, the presence of surface termination groups is responsible for the hydrophilicity and excellent reactivity of MXene, which also facilitates stable colloidal solution preparation in aqueous medium and different solvents ${ }^{[163-165]}$. Similar to the above-mentioned metal compounds and MOFs, MXenes have also been integrated into various elastic substrates for WSCs via coating ${ }^{[16,167]}$, chemical and physical vapor deposition ${ }^{[168,169]}$, and printing $^{[170-173]}$.

For instance, Wang et al. ${ }^{[13]}$ developed a tailorable and foldable solid-state asymmetric supercapacitor through one-step scalable chemical oxidization and $\mathrm{Ti}_{3} \mathrm{C}_{2} \mathrm{~T}_{\mathrm{x}}$ MXene ink painting of $\mathrm{N}$-doped carbon fiber textile (NCFT) substrate. By regulating the oxidization time and MXene loading, the active layer of MXenedecorated NCFT (MNCFT) and O-functionalized NCFT (ONCFT) electrodes analogously presented tight 
skin structure, avoiding the risk of active materials detaching from the support during mechanical deformation. The as-prepared MNCFT/ONCFT supercapacitor possessed high electrochemical performance (extended voltage window of $1.6 \mathrm{~V}$, high areal energy density of $277.3 \mu \mathrm{Wh} \mathrm{cm}^{-2}$, and $90 \%$ capacitance retention after 30,000 cycles) and could be tailored into suitable size or shape for conformable integration without impairing its performance [Figure 14A-G]. However, to ensure the electrochemical properties of the as-prepared MXene-carbon fiber textile electrodes, the textile had to be coated with a large amount of MXenes. Therefore, it remains challenging to convert the electrical insulative textiles into highly conductive textiles at low MXene loading. Some studies tried to resolve this issue by combining MXenes with conductive polymer ${ }^{[174,175]}$ or carbon-based materials ${ }^{[176-178]}$. Zheng et al..$^{[179]}$ reported a novel and facile vapor-phase polymerization and spray-coating strategy to produce PEDOT/MXene-decorated cotton fabrics (PMF). The condensed PEDOT contributed continuous electron pathways and pseudocapacitance to improve both the electrical conductivity and electrochemical performance of textiles [Figure $14 \mathrm{H}$ and I]. Additionally, in addition to combining with conductivity polymer and carbon-based materials, the aforementioned metal compounds and MOFs were integrated to further improve the electrochemical performance of MXene-based electrodes of WSCs ${ }^{[180-182]}$. Although the above studies of flexible textiles make metal-based materials a promising electrode for WSCs, it remains a big challenge to fabricate soft electrodes with the ability to deform into arbitrary shapes with the human body under the moving condition and without sacrificing the electrochemical performance of WSCs. Novel strategies for producing low-cost, environment-friendly, and soft composite materials also need to be further developed.

\section{SOLID-STATE ELECTROLYTES FOR WSCS}

Aqueous electrolytes such as salt, acid, and alkaline solutions are still the most commonly used electrolytes for commercial SCs ${ }^{[10,11]}$. However, these aqueous electrolytes are unable to adapt to WSCs because of the leakage of toxic component when dynamically deformed or implanted into the human body. Thus, solid electrolytes have attracted lots of attention ${ }^{[1,183-185]}$. Hydrogel-based electrolytes are the most studied solid electrolytes as both electrolytes and separators for all-solid-state SCs. Their typical swollen porous structure with absorbed aqueous endows them with high ionic conductivity and less electrolyte leakage possibility. In addition to their highly flexible and stretchable nature, hydrogel-based electrolytes are becoming the most indispensable unit for the rapid development of portable/wearable electrical devices.

PVA is the most widely used solid electrolyte due to its high structural integrity and good mechanical properties ${ }^{[186-191]}$. Moreover, the easy gelation process and the large water content absorbed in the PVA polymer matrix also helps it offer proper ionic conductivity, which makes it widely used in WSCs ${ }^{[192-194]}$ and stretchable SCs ${ }^{[195,196]}$. In addition to PVA hydrogel electrolytes, polyacrylic acid (PAA) ${ }^{[197-199]}$ and natural polymers, such as gelatin ${ }^{[200-202]}$, $\operatorname{lignin}^{[203,204]}$, and cellulose ${ }^{[205,206]}$, were also developed to further improve the high retention of water in the polymer matrix to make sure the ionic conductivity ${ }^{[183,207]}$. For example, crosslinked networks of lignin-based hydrogel electrolytes showing high ionic conductivity $\left(10.4 \mathrm{mS} \mathrm{cm}^{-1}\right.$ at room temperature) and mechanical integrity (swelling capability of 532\% water) were prepared for free-standing, flexible SCs ${ }^{[203]}$. Additionally, these biopolymer-based electrolytes could also be applied in implantable devices, because of their unique merits such as good compactivity, biodegradability, naturel abundance, and sustainability ${ }^{[46,208]}$.

Recently, along with the appearance of smart SCs, self-healable electrolytes are also being investigated to fulfill the demands of self-healable SCs. A smart ionic conductive and self-healable sodium alginate/polymethylacrylic acid hydrogel electrolyte (SPMA-Zn: $\mathrm{ZnSO}_{4}$ ) was developed for flexible hybrid supercapacitors. The as-prepared SPMA-Zn exhibits an excellent self-healing ability and can recover its electrochemical performance after multiple mechanical damages. The assembled supercapacitor displays 

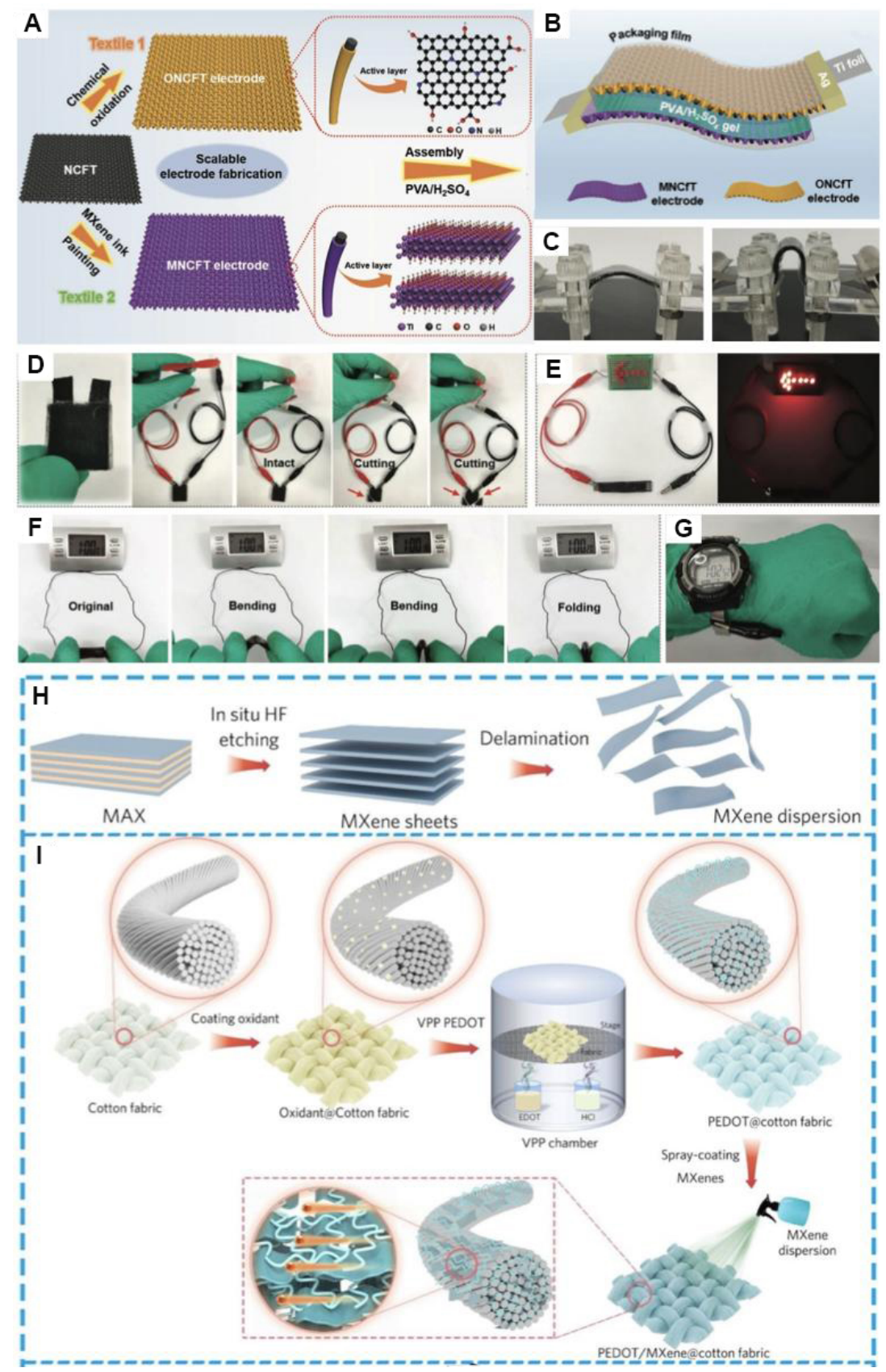

Figure 14. (A) Schematic illustration of the scalable synthesis protocol of the positive ONCFT electrode and negative MNCFT electrode. (B) The assembled MNCFT/ONCFT ASC. (C) Photographs of an MNCFT/ONCFT ASC at different bending angles of 45 and 180. (D) A square MNCFT/ONCFT ASC $(2 \mathrm{~cm} \times 2 \mathrm{~cm})$ normally powers a fan under different damage states. (E) Photograph of two MNCFT/ONCFT ASCs connected in series which can drive an indicating arrow containing 10 red LED lights. (F) Images of an MNCFT/ONCFT ASC driving an electronic watch at different deformable status. (G) Wearable exhibition of an energy storage watchband ${ }^{[13]}$. $(\mathrm{H})$ Schematic illustration of the fabrication of $\mathrm{Ti}_{3} \mathrm{C}_{2} \mathrm{~T}_{\mathrm{x}} \mathrm{MXene}$. (I) Fabrication of PMFs ${ }^{[179]}$. MNCFT: MXene-decorated $\mathrm{N}$-doped carbon fiber textile; ONCFT: O-functionalized N-doped carbon fiber textile.

excellent electrochemical performance with a wide and stable working voltage range of 0-2.2 $\mathrm{V}$, high energy density of $164.13 \mathrm{Wh} \mathrm{kg}^{-1}$ at the power density of $1283.44 \mathrm{Wh} \mathrm{kg}^{-1}$, and good stability with a capacity retention of $95.3 \%$ after 5000 charge-discharge cycles at $10 \mathrm{~A} \mathrm{~g} \mathrm{~g}^{-1[209]}$. However, self-healable is a passive process that requires meticulous manual alignment of electrodes and electrolytes, and sometimes even 
requires external stimuli to ensure more effective healing. The mechanical strength is usually greatly deteriorated and thus brings the risks of performance decay and device failure. This is still a big challenge that needs to be overcome to be further applied in smart WSCs.

Thermal stability of hydrogel electrolytes is also a research challenge because of the polymer/monomer nature and the aqueous electrolyte contained within the hydrogels. Recently, all-temperature hydrogel electrolytes have also been developed to further adapt to harsh environments, especially in severe cold and hot regions, in which WSCs need to work $^{[192,208,210-213]}$. Peng et al. ${ }^{[214]}$ prepared a phosphoric acid/chitosan/PAA (CS-PAAm) hydrogel electrolyte. The as-prepared CS-PAAm hydrogels possess extremely high ionic conductivity and wide range of temperature tolerance from -60 to $100{ }^{\circ} \mathrm{C}$. Additionally, this electrolyte also possesses good adhesiveness, toughness and flexibility, which is also important to further apply it for WSCs. The realization of all temperature self-healing hydrogel electrolytes could promote the development of existing WSCs.

\section{ALL-IN-ONE WEARABLE SUPERCAPACITOR DEVICES}

The most common way to fabricate all-in-one WSCs, whose structure is different from the traditional sandwich structure, is to deposit or in situ grow conductive polymers ${ }^{[212,215,216]} /$ active materials $^{[217,218]}$ on both the upper and lower side of a multifunctional hydrogel electrolyte. For example, Wang et al. ${ }^{[219]}$ produced an all-in-one supercapacitor by in situ growing PANi on high-strength PVA hydrogels [Figure 15A-D]. The asprepared all-in-one SC also had high electrochemical properties (the highest volumetric capacitance is 2220 $\mathrm{mF} \mathrm{cm}{ }^{-3}$ at an aniline concentration of $0.5 \mathrm{~mol} \mathrm{~L}^{-1}$, the capacitance remains $90 \%$ after 7000 cycles, and energy density and areal power density are $42 \mu \mathrm{Wh} \mathrm{cm} \mathrm{cm}^{-2}$ and $160 \mu \mathrm{W} \mathrm{cm} \mathrm{cm}^{-2}$, respectively). Furthermore, the mechanical properties including flexibility, toughness, and stretchability are all dependent on the hydrogel electrolyte.

Hence, based on such investigation, all-in-one SCs with specific functions were also developed along with the development of hydrogel electrolytes. Self-healing, fatigue-resistant, and self-recovering all-in-one supercapacitors were produced through PANi-decorated supramolecular PVA/poly(N-hydroxyethyl acrylamide) (PVA/PHEA) hydrogel electrolyte ${ }^{[212]}$. The multiple hydrogen bonding between PVA and PHEA and the ionic interaction among PANi not only endow excellent mechanical properties (tensile strength of $1.07 \mathrm{MPa}$ and tearing energy of $2492 \mathrm{~J} \mathrm{~m}^{-2}$ ) but also facilitate the transfer of charge and ions, which enhances the good electrochemical performance of the as-prepared all-in-one SC $\left(98 \mathrm{mF} \mathrm{cm}^{-2}\right.$ at current density of $0.2 \mathrm{~mA} \mathrm{~cm}{ }^{-2}$ ). Inspired by the temperature-tolerance hydrogel electrolytes, anti-freezing all-in-one SCs were also developed by one-step in situ growth of PANi onto a hydrogel electrolyte from cross-linked PAM networks soaked in ethylene glycol/water/ $\mathrm{H}_{2} \mathrm{SO}_{4}{ }^{[220]}$. The as-prepared supercapacitors possess high mechanical (stretchability of $200 \%$ at $-30{ }^{\circ} \mathrm{C}$ and can be repeatedly stretched for 100 cycles without significant capacitance loss) and electrochemical properties (91.7\% capacitance retention after 100,000 cycles at $\left.-30^{\circ} \mathrm{C}\right)$.

To further increase the skin-friendliness and safety of all-in-one SCs, moderate acids and biodegradable or natural polymers were applied in the investigation of all-in-one supercapacitors. All-in-one configured flexible and autonomously self-healable supercapacitors were prepared by in situ polymerizing and depositing rGO and PANi onto both sides of the tannic acid-treated gelatin mathacrylate and cellulose hydrogels ${ }^{[221]}$. The as-prepared all-in-one supercapacitor not only has good electrochemical performance (the volume capacitance, energy density, and power density of the as-prepared SC reached $1861 \mathrm{mF} \mathrm{cm}^{-3}$, $20.65 \mathrm{~mW} \mathrm{~cm}^{-3}$, and $595.59 \mathrm{mWh} \mathrm{cm}^{-3}$, respectively) and mechanical properties but also give inspiration to the further study of biocompatible or implantable SCs. However, the stretchability, especially the 


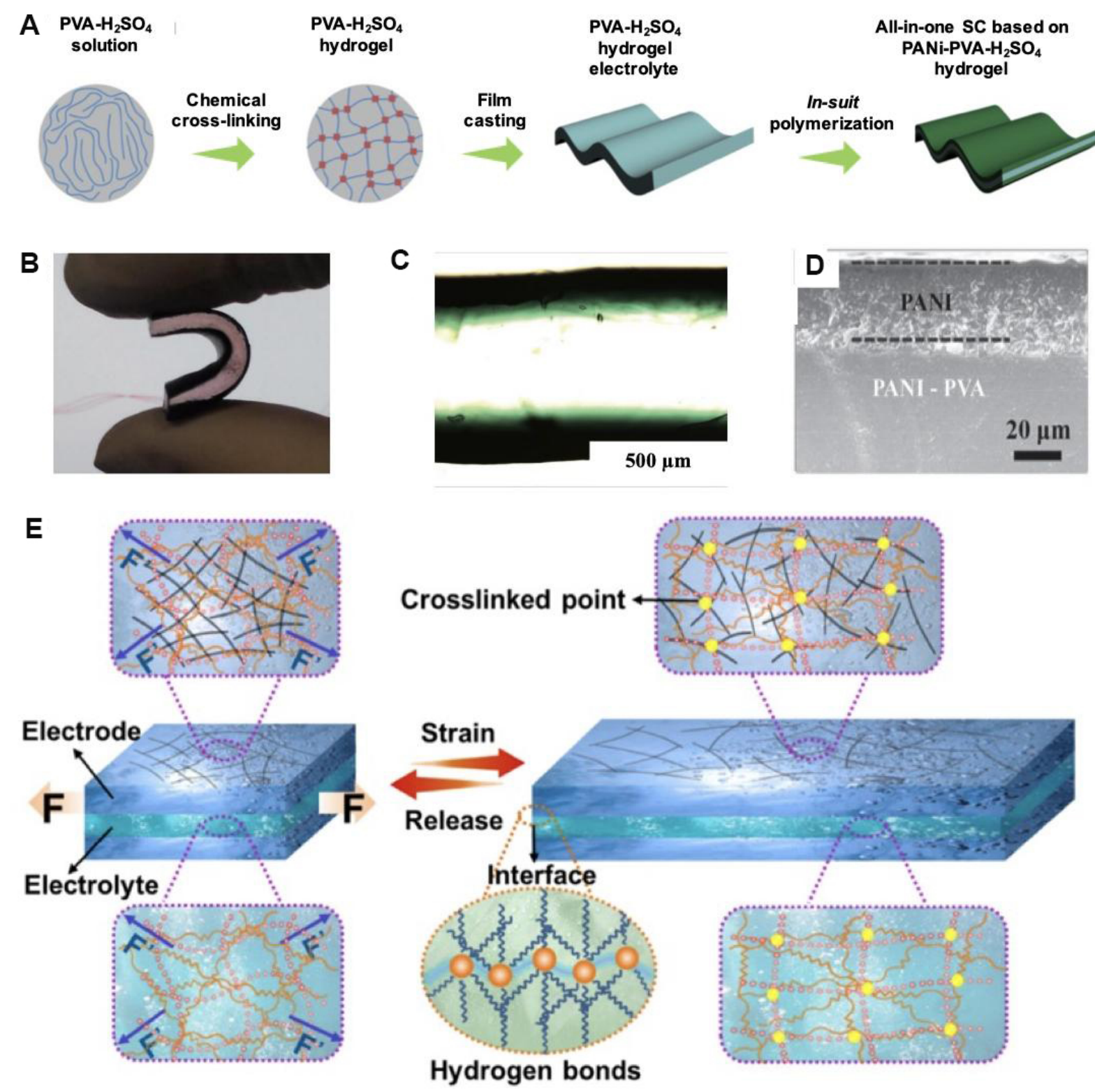

Figure 15. (A) Schematic of the device preparation process of SCs based on a single hydrogel electrolyte. (B) Image of a PANi-PVA SC. (C) Optical microscopy cross-section image of a PANi-PVA SC. (D) Cross-sectional SEM image of a PANi SC ${ }^{[219]}$. (E) Schematic illustration of the stable network structure of an integrated all-hydrogel supercapacitor during the stress-strain process (F: force). (B-G) Electrochemical stability of an all-hydrogel supercapacitor under variant deformations ${ }^{[222]}$.

compressibility, of the aforementioned assembled all-in-one supercapacitors is still limited because of the different mechanical properties of the non-hydrogel electrode and hydrogel electrolytes. Moreover, the energy density and power density still need to be further improved.

To overcome the mismatch of the mechanical properties of the different electrodes and electrolytes in all-inone supercapacitors, all hydrogel-based all-in-one supercapacitors were recently reported. This supercapacitor consisted of hydrogel electrode (polyacrylamide/sodium alginate dual-network hydrogel with additional CNT and PEDOT:PSS) and the same hydrogel electrolyte (polyacrylamide/sodium alginate dual-network hydrogel with salt/redox couple) [Figure 15E] ${ }^{[222]}$. Because of this specific structure and the 
same hydrogel matrix for both electrode and electrolyte, the as-prepared supercapacitor possesses high stretchability and compressibility, strong self-adhesion, and good electrochemical performance (maintain areal capacitance of $128 \mathrm{mF} \mathrm{cm}^{-2}$ at $1 \mathrm{~mA} \mathrm{~cm}^{-2}$, energy density of $3.6 \mu \mathrm{Wh} \mathrm{cm}^{-2}$, and stable energy output). This study, combining the traditional sandwich structure of SCs and the newly developed functional hydrogel electrodes, inspires a new strategy to develop WSCs, which can inherent the multiple functions of hydrogels to match the demands of wearable or implantable bioelectronics.

\section{CHALLENGES AND FUTURE PROSPECTS}

In this report, we discuss the up-to-date advancements in soft electrode materials and their synthesis approaches for WSCs, focusing on the importance of, as well as challenges involved in, endowing supercapacitors with multifunctional properties for wearable electronics. Heretofore, the development of soft materials for supercapacitors that can be deformed into arbitrary shapes and adapt to random musculoskeletal deformations in the human body is still a considerable challenge. The few operational multifunctional SCs that have been reported to date remain largely limited in terms of accommodating external wearable electronics. The development of some kinds of functional SCs such as hydrogel- and degradable material-based SCs satisfying the requirements for next-generation smart wearable or implantable bioelectronics, e.g., tactile sensors, implantable nervous sensors, electrophysiology sensors, and feedback stimulators, remains an arduous task, owing to the complicated physiological environment of the human body.

The electrochemical performance and advantageous characteristics of WSCs, including their areal, volume, and mass capacitance, cycle stability, rate capability, energy density, and power density, are usually determined by their behavior under normal, bent, twisted, or compressed shape conditions. The majority of WSCs that have been developed to date possess multifunctionality features, albeit sacrificing their electrochemical performance to some extent. More importantly, the stable energy output of these devices can hardly be guaranteed under dynamic deformation conditions; furthermore, the majority of studies to date have tested their electrochemical performance under static conditions. Therefore, ensuring the high electrochemical performance of WSCs, especially in terms of energy/power density, while preserving their multifunctionality features under physiological conditions, is probably the most significant challenge. Moreover, washable, breathable, wear-resistant, and comfortable properties, as well as a robust and nontoxic solid-state electrolyte, for WSCs are also needed to be seriously considered to fulfill the demand of everyday use. The stable electrochemical performance of WSCs after repeated washing is also the main limitation in the commercial devices.

Using existing electrode materials and flexible substrate as a starting point for satisfying their energy demand, the development of WSCs exhibits a promising future. Various pathways to design novel materials and device structures still need to reach the desired electrochemical performance and functionality of WSCs. Herein, we discuss the possible future directions for the development of WSCs for wearable and implantable bioelectronics.

\section{Hydrogel electrodes combining conductive fillers}

Embedding conductive fillers which perform EDLC or pseudocapacitance behavior in an existing nonconductive hydrogel matrix is also an effective strategy for fabricating conductive soft hydrogels, which could also be potential soft electrodes for WSCs. Han et al. ${ }^{[223]}$ prepared a dual-network structure multifunctional polyvinyl alcohol (PVA)/CNT-cellulose composite hydrogel electrode for WSCs. The asprepared solid-state supercapacitor obtains $117.1 \mathrm{~F} \mathrm{~g}^{-1}$ and capacitance retention of $96.4 \%$ after 1000 cycles. PVA has excellent hydrophilicity, solubility, biocompatibility, and non-toxicity properties ${ }^{[224]}$. Therefore, 
PVA-based conductive hybrid hydrogels could inherit the mechanical properties of the PVA matrix and present special features, such as compressivity, toughness, and thermal stability, which are also desired for further intelligent WSCs ${ }^{[225,226]}$. To date, except PVA-based hydrogel electrodes for SCs, few works focus on the other polymer-based hydrogel electrodes for WSCs; thus, our group designed a series of self-adhesive, tough, and conductive polyacrylamide hydrogels ${ }^{[227-231]}$ which could be further applied as soft electrodes for WSCs. In these previous studies, polydopamine (PDA) was combined with the hydrogels, which endowed the hydrogels with good self-adhesiveness inspired by the mussel ${ }^{[232]}$. Self-adhesiveness makes hydrogels guarantee comfortable wear experience, reliable and comfortable contact with tissue, and reduced interface resistance for the stable signal detection when applied in bioelectronics ${ }^{[23,234]}$. Furthermore, PDA, as a quinone-rich polymer, has already been applied in energy storage materials due to the redox behavior of quinone ${ }^{[235-237]}$. rGO has also been extensively applied as electrodes for $\mathrm{SCs}^{[238,239]}$. Based on the designed component of PDA and rGO, these conductive and self-adhesive hydrogels could also be potential candidates for functional WSCs. Other materials with pseudocapacitance behavior such as conductive polymers (PEDOT, PANi, and PPy), MOFs, and metal compounds have also been applied, which not only improve their electrochemical performance but also endow the electrodes with repeatable, long-term mussel-inspired self-adhesiveness ${ }^{[231]}$. These results also inspired our following study on multifunctional hydrogel electrodes for future intelligent multifunctional SCs in wearable and implantable bioelectronics.

\section{Multifunctional soft electrodes for supercapacitors}

One of the primary aspects of modern supercapacitor development is to accommodate the power needs of the soft bioelectronic devices used to monitor human health conditions. To successfully adapt to this unique physiological environment, soft electrodes with the ability to be stretched, compressed, bent, twisted, and deformed into arbitrary shapes must be further studied and developed.

Self-adhesion reduces the interfacial resistance between the electrode and the electrolyte, while further guaranteeing the stable, accurate, and simultaneous sensing of multiple stimuli by allowing the direct and robust adhesion of WSCs to human skin or other tissues under tensile, compressive, or twisting stress conditions ${ }^{[240-242]}$. In a dynamic/random motion environment, flexible, stretchable, tough, and self-adhesive bioelectronics are highly desired to ensure the conformal contact between the device and the biological tissue, a factor which critically affects their performance. Hence, soft materials such as hydrogel-based electrodes with self-adhesive properties are highly desirable for matching these advanced bioelectronics.

\section{Implantable or biodegradable in vivo WSCs}

Implantable or biodegradable WSCs are completely or partially embedded into the human or animal body and remain inside to match or power other bioelectronics and complete special tasks. The development of implantable SCs is an arduous task that must address multiple issues, such as the aspects of biocompatibility and biostability. For the long-term implantation of SCs, power output stability is another important factor that needs to be considered. At the same time, several important material properties, such as mechanical strength and conductivity, are significantly compromised by the swelling of materials under the water-rich human physiological solutions. Thus, materials with superior biocompatibility and long-term biostability must be designed to obtain functional electrode materials. To achieve power output stability, certain techniques that rely on harvesting energy from movement, chemical reactions, and ion-related changes in the human body also provide inspiration for the design of sophisticated implantable SCs ${ }^{[6,243,244]}$. Furthermore, some types of SCs consisting of environmentally and biologically degradable byproducts can completely dissolve in vivo after performing their special functions ${ }^{[46]}$. Therefore, biodegradable hydrogels need to be designed for functional biodegradable SCs. 


\section{Environmentally tolerant WSCs}

Environmental tolerance is of great significance for WSCs, especially for applications in harsh environments. Conventional supercapacitors can operate in the temperature range from -40 to $200{ }^{\circ} \mathrm{C}^{[245,246]}$. The temperature tolerance of the devices is mainly dependent on the temperature tolerance of the electrode materials and hydrogel electrolytes. Thus, improving the temperature tolerance and stability of electrodes without compromising their other functionalities also constitutes a considerable challenge in designing WSCs with excellent durability and adaptability to harsh and complex environments.

\section{DECLARATIONS}

\section{Author's contributions}

Designed and wrote the manuscript: Jiang L

Provided theoretical support: Yuan L, Zhang Q

Organized references: Wang W

\section{Availability of data and materials}

Not applicable.

\section{Financial support and sponsorship}

This work was financially supported by National Natural Science Foundation of China (No. 5197227).

\section{Conflicts of interest}

All authors declared that there are no conflicts of interest.

\section{Ethical approval and consent to participate}

Not applicable.

\section{Consent for publication}

Not applicable.

\section{Copyright}

(c) The Author(s) 2021.

\section{REFERENCES}

1. Xue Q, Sun J, Huang Y, et al. Recent progress on flexible and wearable supercapacitors. Small 2017;13:1701827. DOI PubMed

2. Muralee gopi CV, Vinodh R, Sambasivam S, Obaidat IM, Kim H. Recent progress of advanced energy storage materials for flexible and wearable supercapacitor: From design and development to applications. Journal of Energy Storage 2020;27:101035. DOI

3. Fu Y, Cai X, Wu H, et al. Fiber supercapacitors utilizing pen ink for flexible/wearable energy storage. Adv Mater 2012;24:5713-8. DOI PubMed

4. Sumboja A, Liu J, Zheng WG, Zong Y, Zhang H, Liu Z. Electrochemical energy storage devices for wearable technology: a rationale for materials selection and cell design. Chem Soc Rev 2018;47:5919-45. DOI PubMed

5. Li H, Tang Z, Liu Z, Zhi C. Evaluating flexibility and wearability of flexible energy storage devices. Joule 2019;3:613-9. DOI

6. Taube Navaraj W, García Núñez C, Shakthivel D, et al. Nanowire FET based neural element for robotic tactile sensing skin. Front Neurosci 2017;11:501. DOI PubMed PMC

7. Nazari A, Farhad S. Heat generation in lithium-ion batteries with different nominal capacities and chemistries. Appl Therm Eng 2017;125:1501-17. DOI

8. Dong L, Xu C, Li Y, et al. Flexible electrodes and supercapacitors for wearable energy storage: a review by category. J Mater Chem A 2016;4:4659-85. DOI

9. Yun TG, Park M, Kim DH, et al. All-transparent stretchable electrochromic supercapacitor wearable patch device. ACS Nano 2019;13:3141-50. DOI PubMed

10. Yu L, Chen GZ. Ionic liquid-based electrolytes for supercapacitor and supercapattery. Front Chem 2019;7:272. DOI PubMed PMC

11. Zhong C, Deng Y, Hu W, Qiao J, Zhang L, Zhang J. A review of electrolyte materials and compositions for electrochemical supercapacitors. Chem Soc Rev 2015;44:7484-539. DOI PubMed

12. Xu T, Yang D, Zhang S, Zhao T, Zhang M, Yu Z. Antifreezing and stretchable all-gel-state supercapacitor with enhanced capacitances established by graphene/PEDOT-polyvinyl alcohol hydrogel fibers with dual networks. Carbon 2021;171:201-10. DOI 
13. Wang Y, Wang X, Li X, et al. A high-performance, tailorable, wearable, and foldable solid-state supercapacitor enabled by arranging pseudocapacitive groups and MXene flakes on textile electrode surface. Adv Funct Mater 2021;31:2008185. DOI

14. Zhao J, Lu H, Zhang Y, et al. Direct coherent multi-ink printing of fabric supercapacitors. Sci Adv 2021;7:eabd6978. DOI PubMed PMC

15. Ji X, Wang Q, Yu M, et al. All-in-one energy storage devices supported and interfacially cross-linked by gel polymeric electrolyte. Energy Storage Materials 2021;37:587-97. DOI

16. Sheng H, Zhou J, Li B, et al. A thin, deformable, high-performance supercapacitor implant that can be biodegraded and bioabsorbed within an animal body. Sci Adv 2021;7:eabe3097. DOI PubMed PMC

17. Jiang L, Mei X, Gan D, et al. Hybrid transition-metal oxide and nitride@N-doped reduced graphene oxide electrodes for highperformance, flexible, and all-solid-state supercapacitors. Chemistry 2021;27:5761-8. DOI PubMed

18. Zhang L, Hu X, Wang Z, Sun F, Dorrell DG. A review of supercapacitor modeling, estimation, and applications: a control/management perspective. Renew Sust Energ Rev 2018;81:1868-78. DOI

19. Jeong JW, Shin G, Park SI, Yu KJ, Xu L, Rogers JA. Soft materials in neuroengineering for hard problems in neuroscience. Neuron 2015;86:175-86. DOI PubMed

20. Raza W, Ali F, Raza N, et al. Recent advancements in supercapacitor technology. Nano Energy 2018;52:441-73. DOI

21. Jiang L, Luo D, Zhang Q, et al. Electrochemical performance of free-standing and flexible graphene and $\mathrm{TiO}_{2}$ composites with different conductive polymers as electrodes for supercapacitors. Chemistry 2019;25:7903-11. DOI PubMed

22. Zhang LL, Zhao XS. Carbon-based materials as supercapacitor electrodes. Chem Soc Rev 2009;38:2520-31. DOI PubMed

23. Da Silva LM, Cesar R, Moreira CM, et al. Reviewing the fundamentals of supercapacitors and the difficulties involving the analysis of the electrochemical findings obtained for porous electrode materials. Energy Storage Materials 2020;27:555-90. DOI

24. Chen Z, Wang X, Xue B, et al. Rice husk-based hierarchical porous carbon for high performance supercapacitors: the structureperformance relationship. Carbon 2020;161:432-44. DOI

25. Wang Y, Chen F, Liu Z, et al. A highly elastic and reversibly stretchable all-polymer supercapacitor. Angew Chem 2019;131:158548. DOI PubMed

26. Liu R, Zhou A, Zhang X, et al. Fundamentals, advances and challenges of transition metal compounds-based supercapacitors. Chem Eng $J$ 2021;412:128611. DOI

27. Nguyen T, Montemor MF. Metal oxide and hydroxide-based aqueous supercapacitors: from charge storage mechanisms and functional electrode engineering to need-tailored devices. Adv Sci (Weinh) 2019;6:1801797. DOI PubMed PMC

28. Naskar P, Maiti A, Chakraborty P, Kundu D, Biswas B, Banerjee A. Chemical supercapacitors: a review focusing on metallic compounds and conducting polymers. J Mater Chem A 2021;9:1970-2017. DOI

29. Lim HR, Kim HS, Qazi R, Kwon YT, Jeong JW, Yeo WH. Advanced soft materials, sensor integrations, and applications of wearable flexible hybrid electronics in healthcare, energy, and environment. Adv Mater 2020;32:e1901924. DOI PubMed

30. Kazem N, Hellebrekers T, Majidi C. Soft multifunctional composites and emulsions with liquid metals. Adv Mater 2017;29:1605985. DOI PubMed

31. Kim DH, Lu N, Ma R, et al. Epidermal electronics. Science 2011;333:838-43. DOI PubMed

32. Song Y, Chen H, Chen X, et al. All-in-one piezoresistive-sensing patch integrated with micro-supercapacitor. Nano Energy 2018;53:189-97. DOI

33. Yu C, Masarapu C, Rong J, Wei B, Jiang H. Stretchable supercapacitors based on buckled single-walled carbon-nanotube macrofilms. Adv Mater 2009;21:4793-7. DOI PubMed

34. Lacour SP, Courtine G, Guck J. Materials and technologies for soft implantable neuroprostheses. Nat Rev Mater 2016:1. DOI

35. Rivnay J, Wang H, Fenno L, Deisseroth K, Malliaras GG. Next-generation probes, particles, and proteins for neural interfacing. Sci Adv 2017;3:e1601649. DOI PubMed PMC

36. Yang Z, Deng J, Chen X, Ren J, Peng H. A highly stretchable, fiber-shaped supercapacitor. Angew Chem 2013;125:13695-9. DOI PubMed

37. An T, Cheng W. Recent progress in stretchable supercapacitors. J Mater Chem A 2018;6:15478-94. DOI

38. Li X, Li H, Fan X, Shi X, Liang J. 3D-printed stretchable micro-supercapacitor with remarkable areal performance. Adv Energy Mater 2020;10:1903794. DOI

39. Zheng Z, Jin J, Dong JC, et al. Unusual sonochemical assembly between carbon allotropes for high strain-tolerant conductive nanocomposites. ACS Nano 2019;13:12062-9. DOI PubMed PMC

40. Zhi J, Zhao W, Liu X, Chen A, Liu Z, Huang F. Highly conductive ordered mesoporous carbon based electrodes decorated by 3D graphene and 1D silver nanowire for flexible supercapacitor. Adv Funct Mater 2014;24:2013-9. DOI

41. Javed MS, Shaheen N, Hussain S, et al. An ultra-high energy density flexible asymmetric supercapacitor based on hierarchical fabric decorated with 2D bimetallic oxide nanosheets and MOF-derived porous carbon polyhedra. J Mater Chem A 2019;7:946-57. DOI

42. Sheberla D, Bachman JC, Elias JS, Sun CJ, Shao-Horn Y, Dincă M. Conductive MOF electrodes for stable supercapacitors with high areal capacitance. Nat Mater 2017;16:220-4. DOI PubMed

43. Yan J, Ren CE, Maleski K, et al. Flexible MXene/Graphene films for ultrafast supercapacitors with outstanding volumetric capacitance. Adv Funct Mater 2017;27:1701264. DOI

44. Sim HJ, Choi C, Lee DY, et al. Biomolecule based fiber supercapacitor for implantable device. Nano Energy 2018;47:385-92. DOI

45. He S, Hu Y, Wan J, et al. Biocompatible carbon nanotube fibers for implantable supercapacitors. Carbon 2017;122:162-7. DOI

46. Tian W, Li Y, Zhou J, et al. Implantable and biodegradable micro-supercapacitor based on a superassembled three-dimensional network Zn@PPy hybrid electrode. ACS Appl Mater Interfaces 2021;13:8285-93. DOI PubMed 
47. wu J, Xia M, Zhang X, et al. Hierarchical porous carbon derived from wood tar using crab as the template: performance on supercapacitor. J Power Sources 2020;455:227982. DOI

48. Tan S, Kraus TJ, Li-oakey KD. Understanding the supercapacitor properties of electrospun carbon nanofibers from powder river basin coal. Fuel 2019;245:148-59. DOI

49. Gopalakrishnan A, Badhulika S. Sulfonated porous carbon nanosheets derived from oak nutshell based high-performance supercapacitor for powering electronic devices. Renewable Energy 2020;161:173-83. DOI

50. Jiang L, Ren Z, Chen S, et al. Bio-derived three-dimensional hierarchical carbon-graphene- $\mathrm{TiO}_{2}$ as electrode for supercapacitors. $S c i$ Rep 2018;8:4412. DOI PubMed PMC

51. Pumera M. Graphene-based nanomaterials and their electrochemistry. Chem Soc Rev 2010;39:4146-57. DOI PubMed

52. Neto AH, Guinea F, Peres NMR, Novoselov KS, Geim AK. The electronic properties of graphene. Rev Mod Phys 2009;81:109-62. DOI

53. Moon IK, Lee J, Ruoff RS, Lee H. Reduced graphene oxide by chemical graphitization. Nat Commun 2010;1:73. DOI PubMed

54. Stoller MD, Park S, Zhu Y, An J, Ruoff RS. Graphene-based ultracapacitors. Nano Lett 2008;8:3498-502. DOI PubMed

55. Compton OC, Nguyen ST. Graphene oxide, highly reduced graphene oxide, and graphene: versatile building blocks for carbon-based materials. Small 2010;6:711-23. DOI PubMed

56. Chen H, Müller MB, Gilmore KJ, Wallace GG, Li D. Mechanically strong, electrically conductive, and biocompatible graphene paper. Adv Mater 2008;20:3557-61. DOI

57. Liu F, Song S, Xue D, Zhang H. Folded structured graphene paper for high performance electrode materials. Adv Mater 2012;24:1089-94. DOI PubMed

58. Pei S, Zhao J, Du J, Ren W, Cheng H. Direct reduction of graphene oxide films into highly conductive and flexible graphene films by hydrohalic acids. Carbon 2010;48:4466-74. DOI

59. Xu Y, Lin Z, Huang X, Liu Y, Huang Y, Duan X. Flexible solid-state supercapacitors based on three-dimensional graphene hydrogel films. ACS Nano 2013;7:4042-9. DOI PubMed

60. Xiong Z, Liao C, Han W, Wang X. Mechanically tough large-area hierarchical porous graphene films for high-performance flexible supercapacitor applications. Adv Mater 2015;27:4469-75. DOI PubMed

61. Xu Y, Lin Z, Huang X, Wang Y, Huang Y, Duan X. Functionalized graphene hydrogel-based high-performance supercapacitors. Adv Mater 2013;25:5779-84. DOI PubMed

62. Yuan S, Fan W, Jin Y, Wang D, Liu T. Free-standing flexible graphene-based aerogel film with high energy density as an electrode for supercapacitors. Nano Materials Science 2021;3:68-74. DOI

63. Yu P, Zhao X, Huang Z, Li Y, Zhang Q. Free-standing three-dimensional graphene and polyaniline nanowire arrays hybrid foams for high-performance flexible and lightweight supercapacitors. J Mater Chem A 2014;2:14413-20. DOI

64. Guo X, Bai N, Tian Y, Gai L. Free-standing reduced graphene oxide/polypyrrole films with enhanced electrochemical performance for flexible supercapacitors. J Power Sources 2018;408:51-7. DOI

65. Zou Z, Zhou W, Zhang Y, Yu H, Hu C, Xiao W. High-performance flexible all-solid-state supercapacitor constructed by freestanding cellulose/reduced graphene oxide/silver nanoparticles composite film. Chem Eng J 2019;357:45-55. DOI

66. Liu F, Xie L, Wang L, et al. Hierarchical porous RGO/PEDOT/PANI hybrid for planar/linear supercapacitor with outstanding flexibility and stability. Nanomicro Lett 2020;12:17. DOI PubMed PMC

67. Yu D, Dai L. Self-assembled graphene/carbon nanotube hybrid films for supercapacitors. J Phys Chem Lett 2010;1:467-70. DOI

68. Gao M, Wang P, Jiang L, et al. Power generation for wearable systems. Energy Environ Sci 2021;14:2114-57. DOI

69. Manjakkal L, Navaraj WT, Núñez CG, Dahiya R. Graphene-graphite polyurethane composite based high-energy density flexible supercapacitors. Adv Sci (Weinh) 2019;6:1802251. DOI PubMed PMC

70. Atta MM, Abdel Maksoud MIA, Sallam OI, Awed AS. Gamma irradiation synthesis of wearable supercapacitor based on reduced graphene oxide/cotton yarn electrode. J Mater Sci: Mater Electron 2021;32:3688-98. DOI

71. El-Kady MF, Strong V, Dubin S, Kaner RB. Laser scribing of high-performance and flexible graphene-based electrochemical capacitors. Science 2012;335:1326-30. DOI PubMed

72. Afroj S, Tan S, Abdelkader AM, Novoselov KS, Karim N. Highly conductive, scalable, and machine washable graphene-based Etextiles for multifunctional wearable electronic applications. Adv Funct Mater 2020;30:2000293. DOI

73. Tran T, Dutta NK, Roy Choudhury N. Graphene-based inks for printing of planar micro-supercapacitors: a review. Materials (Basel) 2019;12:978. DOI PubMed PMC

74. Le LT, Ervin MH, Qiu H, Fuchs BE, Lee WY. Graphene supercapacitor electrodes fabricated by inkjet printing and thermal reduction of graphene oxide. Electrochem Commun 2011;13:355-8. DOI

75. Li H, Liu S, Li X, Wu Z, Liang J. Screen-printing fabrication of high volumetric energy density micro-supercapacitors based on highresolution thixotropic-ternary hybrid interdigital micro-electrodes. Mater Chem Front 2019;3:626-35. DOI

76. Li G, Mo X, Law W, Chan KC. 3D printed graphene/nickel electrodes for high areal capacitance electrochemical storage. $J$ Mater Chem A 2019;7:4055-62. DOI

77. Liu L, Lu J, Long X, et al. 3D printing of high-performance micro-supercapacitors with patterned exfoliated graphene/carbon nanotube/silver nanowire electrodes. Sci China Technol Sci 2021;64:1065-73. DOI

78. Liu J, Ye J, Pan F, Wang X, Zhu Y. Solid-state yet flexible supercapacitors made by inkjet-printing hybrid ink of carbon quantum dots/graphene oxide platelets on paper. Sci China Mater 2019;62:545-54. DOI

79. Sundriyal P, Bhattacharya S. Inkjet-printed electrodes on A4 paper substrates for low-cost, disposable, and flexible asymmetric supercapacitors. ACS Appl Mater Interfaces 2017;9:38507-21. DOI PubMed 
80. Lee S, Choi K, Kim S, Lee S. Wearable supercapacitors printed on garments. Adv Funct Mater 2018;28:1705571. DOI

81. Delekta S, Smith AD, Li J, Östling M. Inkjet printed highly transparent and flexible graphene micro-supercapacitors. Nanoscale 2017;9:6998-7005. DOI PubMed

82. Pham M, Khazaeli A, Godbille-cardona G, Truica-marasescu F, Peppley B, Barz DP. Printing of graphene supercapacitors with enhanced capacitances induced by a leavening agent. J Energy Storage 2020;28:101210. DOI

83. Hyun WJ, Secor EB, Kim C, Hersam MC, Francis LF, Frisbie CD. Scalable, self-aligned printing of flexible graphene microsupercapacitors. Adv Energy Mater 2017;7:1700285. DOI

84. Yang B, Xiong Y, Ma K, Liu S, Tao X. Recent advances in wearable textile-based triboelectric generator systems for energy harvesting from human motion. EcoMat 2020:2. DOI

85. Zhou Y, Wang CH, Lu W, Dai L. Recent advances in fiber-shaped supercapacitors and lithium-ion batteries. Adv Mater 2020;32:e1902779. DOI PubMed

86. Meng Y, Zhao Y, Hu C, et al. All-graphene core-sheath microfibers for all-solid-state, stretchable fibriform supercapacitors and wearable electronic textiles. Adv Mater 2013;25:2326-31. DOI PubMed

87. Liu L, Yu Y, Yan C, Li K, Zheng Z. Wearable energy-dense and power-dense supercapacitor yarns enabled by scalable graphenemetallic textile composite electrodes. Nat Commun 2015;6:7260. DOI PubMed PMC

88. Yuk H, Lu B, Zhao X. Hydrogel bioelectronics. Chem Soc Rev 2019;48:1642-67. DOI PubMed

89. Qu G, Cheng J, Li X, et al. A Fiber Supercapacitor with high energy density based on hollow graphene/conducting polymer fiber electrode. Adv Mater 2016;28:3646-52. DOI PubMed

90. Zhang L, Shi G. Preparation of highly conductive graphene hydrogels for fabricating supercapacitors with high rate capability. $J$ Phys Chem C 2011;115:17206-12. DOI

91. Chen P, Yang J, Li S, et al. Hydrothermal synthesis of macroscopic nitrogen-doped graphene hydrogels for ultrafast supercapacitor. Nano Energy 2013;2:249-56. DOI

92. Lien C, Vedhanarayanan B, Chen J, et al. Optimization of acetonitrile/water content in hybrid deep eutectic solvent for graphene/MoS2 hydrogel-based supercapacitors. Chemical Engineering Journal 2021;405:126706. DOI

93. Kang J, Hwang M, Seong K, Lyu L, Ko D, Piao Y. Three-dimensional nanocomposite of graphene/MWCNT hydrogel grafted with Ni-Co hydroxide nanorods as high-performance electrode for asymmetric supercapacitor. Electrochimica Acta 2020;346:136258. DOI

94. Khazaeli A, Godbille-cardona G, Barz DPJ. A novel flexible hybrid battery-supercapacitor based on a self-assembled vanadiumgraphene hydrogel. Adv Funct Mater 2020;30:1910738. DOI

95. Wu D, Zhong W. A new strategy for anchoring a functionalized graphene hydrogel in a carbon cloth network to support a lignosulfonate/polyaniline hydrogel as an integrated electrode for flexible high areal-capacitance supercapacitors. $J$ Mater Chem A 2019;7:5819-30. DOI

96. Zou Y, Zhang Z, Zhong W, Yang W. Hydrothermal direct synthesis of polyaniline, graphene/polyaniline and N-doped graphene/polyaniline hydrogels for high performance flexible supercapacitors. J Mater Chem A 2018;6:9245-56. DOI

97. Ates M, El-Kady M, Kaner RB. Three-dimensional design and fabrication of reduced graphene oxide/polyaniline composite hydrogel electrodes for high performance electrochemical supercapacitors. Nanotechnology 2018;29:175402. DOI PubMed

98. Chen T, Dai L. Flexible supercapacitors based on carbon nanomaterials. J Mater Chem A 2014;2:10756. DOI

99. Chen H, Zeng S, Chen M, Zhang Y, Li Q. Fabrication and functionalization of carbon nanotube films for high-performance flexible supercapacitors. Carbon 2015;92:271-96. DOI

100. Senokos E, Rana M, Santos C, Marcilla R, Vilatela JJ. Controlled electrochemical functionalization of CNT fibers: structurechemistry relations and application in current collector-free all-solid supercapacitors. Carbon 2019;142:599-609. DOI

101. Lee JA, Shin MK, Kim SH, et al. Ultrafast charge and discharge biscrolled yarn supercapacitors for textiles and microdevices. Nat Commun 2013;4:1970. DOI PubMed

102. Patil B, Ahn S, Yu S, et al. Electrochemical performance of a coaxial fiber-shaped asymmetric supercapacitor based on nanostructured MnO2/CNT-web paper and Fe2O3/carbon fiber electrodes. Carbon 2018;134:366-75. DOI

103. Wang S, Liang Y, Zhuo W, et al. Freestanding polypyrrole/carbon nanotube electrodes with high mass loading for robust flexible supercapacitors. Mater Chem Front 2021;5:1324-9. DOI

104. Mirabedini A, Lu Z, Mostafavian S, Foroughi J. Triaxial carbon nanotube/conducting polymer wet-spun fibers supercapacitors for wearable electronics. Nanomaterials (Basel) 2020;11:3. DOI PubMed PMC

105. Cao C, Zhou Y, Ubnoske S, et al. Highly stretchable supercapacitors via crumpled vertically aligned carbon nanotube forests. $A d v$ Energy Mater 2019;9:1900618. DOI

106. Liu L, Niu Z, Chen J. Design and integration of flexible planar micro-supercapacitors. Nano Res 2017;10:1524-44. DOI

107. Zhang R, Yan K, Palumbo A, Xu J, Fu S, Yang EH. A stretchable and bendable all-solid-state pseudocapacitor with dodecylbenzenesulfonate-doped polypyrrole-coated vertically aligned carbon nanotubes partially embedded in PDMS. Nanotechnology 2019;30:095401. DOI PubMed

108. Pu X, Li L, Liu M, et al. Wearable self-charging power textile based on flexible yarn supercapacitors and fabric nanogenerators. $A d v$ Mater 2016;28:98-105. DOI PubMed

109. Niu Z, Liu L, Zhang L, Zhou W, Chen X, Xie S. Programmable nanocarbon-based architectures for flexible supercapacitors. Adv Energy Mater 2015;5:1500677. DOI

110. Guo T, Zhou D, Liu W, Su J. Recent advances in all-in-one flexible supercapacitors. Sci China Mater 2021;64:27-45. DOI

111. Wang Y, Ding Y, Guo X, Yu G. Conductive polymers for stretchable supercapacitors. Nano Res 2019;12:1978-87. DOI 
112. Shi Y, Peng L, Ding Y, Zhao Y, Yu G. Nanostructured conductive polymers for advanced energy storage. Chem Soc Rev 2015;44:6684-96. DOI PubMed

113. Wang G, Zhang L, Zhang J. A review of electrode materials for electrochemical supercapacitors. Chem Soc Rev 2012;41:797-828. DOI PubMed

114. Wang L, Zhang C, Jiao X, Yuan Z. Polypyrrole-based hybrid nanostructures grown on textile for wearable supercapacitors. Nano Res 2019;12:1129-37. DOI

115. Chu X, Chen G, Xiao X, et al. Air-Stable conductive polymer ink for printed wearable micro-supercapacitors. Small 2021;17:e2100956. DOI PubMed

116. Zhao J, Zhang Y, Zhao X, et al. Direct ink writing of adjustable electrochemical energy storage device with high gravimetric energy densities. Adv Funct Mater 2019;29:1900809. DOI

117. Kayser LV, Lipomi DJ. Stretchable conductive polymers and composites based on PEDOT and PEDOT:PSS. Adv Mater 2019;31:e1806133. DOI PubMed PMC

118. Yang J, Cao Q, Tang X, et al. 3D-printed highly stretchable conducting polymer electrodes for flexible supercapacitors. $J$ Mater Chem A 2021. DOI

119. Wang K, Zhang X, Li C, et al. Flexible solid-state supercapacitors based on a conducting polymer hydrogel with enhanced electrochemical performance. J Mater Chem A 2014;2:19726-32. DOI

120. Shi Y, Pan L, Liu B, et al. Nanostructured conductive polypyrrole hydrogels as high-performance, flexible supercapacitor electrodes. J Mater Chem A 2014;2:6086-91. DOI

121. Ghosh S, Inganäs O. Conducting polymer hydrogels as 3D electrodes: applications for supercapacitors. Adv Mater 1999;11:1214-8. DOI

122. Shown I, Ganguly A, Chen L, Chen K. Conducting polymer-based flexible supercapacitor. Energy Sci Eng 2015;3:2-26. DOI

123. Pan L, Yu G, Zhai D, et al. Hierarchical nanostructured conducting polymer hydrogel with high electrochemical activity. Proc Natl Acad Sci U S A 2012;109:9287-92. DOI PubMed PMC

124. Green RA, Baek S, Poole-Warren LA, Martens PJ. Conducting polymer-hydrogels for medical electrode applications. Sci Technol Adv Mater 2010;11:014107. DOI PubMed PMC

125. Das S, Chakraborty P, Mondal S, Shit A, Nandi AK. Enhancement of energy storage and photoresponse properties of folic acidpolyaniline hybrid hydrogel by in situ growth of Ag nanoparticles. ACS Appl Mater Interfaces 2016;8:28055-67. DOI PubMed

126. Wang Y, Shi Y, Pan L, et al. Dopant-enabled supramolecular approach for controlled synthesis of nanostructured conductive polymer hydrogels. Nano Lett 2015;15:7736-41. DOI PubMed

127. Chen Z, To JWF, Wang C, et al. A three-dimensionally interconnected carbon nanotube-conducting polymer hydrogel network for high-performance flexible battery electrodes. Adv Energy Mater 2014;4:1400207. DOI

128. Zhao Y, Zhang B, Yao B, et al. Hierarchically structured stretchable conductive hydrogels for high-performance wearable strain sensors and supercapacitors. Matter 2020;3:1196-210. DOI

129. Li L, Shi Y, Pan L, Shi Y, Yu G. Rational design and applications of conducting polymer hydrogels as electrochemical biosensors. $J$ Mater Chem B 2015;3:2920-30. DOI PubMed

130. Jiang L, Lu X, Xie C, Wan G, Zhang H, Youhong T. Flexible, free-standing $\mathrm{TiO}_{2}$-graphene-polypyrrole composite films as electrodes for supercapacitors. $J$ Phys Chem C 2015;119:3903-10. DOI

131. Jiang L, Li Y, Luo D, et al. Freestanding RGO- $\mathrm{Co}_{3} \mathrm{O}_{4}$-PPy Composite Films as Electrodes for Supercapacitors. Energy Technol 2019;7:1800606. DOI

132. Jiang L, Song S, Luo D, et al. Plant growth-inspired design of high-performance composite electrode nanostructures for supercapacitors. Materials Today Physics 2020;12:100138. DOI

133. Jia Y, Jiang X, Ahmed A, Zhou L, Fan Q, Shao J. Microfluidic-architected core-shell flower-like $\delta$-MnO2@graphene fibers for high energy-storage wearable supercapacitors. Electrochimica Acta 2021;372:137827. DOI

134. Karami Z, Youssefi M, Raeissi K, Zhiani M. An efficient textile-based electrode utilizing silver nanoparticles/reduced graphene oxide/cotton fabric composite for high-performance wearable supercapacitors. Electrochimica Acta 2021;368:137647. DOI

135. Salman A, Padmajan Sasikala S, Kim IH, et al. Tungsten nitride-coated graphene fibers for high-performance wearable supercapacitors. Nanoscale 2020;12:20239-49. DOI PubMed

136. Yi Y, Yu L, Tian Z, et al. Biotemplated synthesis of transition metal nitride architectures for flexible printed circuits and wearable energy storages. Adv Funct Mater 2018;28:1805510. DOI

137. Peng Z, Huang J, He Q, Tan L, Chen Y. Highly porous $\mathrm{Mn}_{3} \mathrm{O}_{4}$ nanosheets with in situ coated carbon enabling fully screen-printed planar supercapacitors with remarkable volumetric performance. J Mater Chem A 2021;9:4273-80. DOI

138. Hoskins BF, Robson R. Infinite polymeric frameworks consisting of three dimensionally linked rod-like segments. J Am Chem Soc 1989;111:5962-4. DOI

139. Hoskins BF, Robson R. Design and construction of a new class of scaffolding-like materials comprising infinite polymeric frameworks of 3D-linked molecular rods. A reappraisal of the zinc cyanide and cadmium cyanide structures and the synthesis and structure of the diamond-related frameworks [N(CH3)4][CuIZnII(CN)4] and CuI[4, 4', 4'", 4'"'tetracyanotetraphenylmethane]BF4.xC6H5NO2. J Am Chem Soc 1990;112:1546-54. DOI

140. Shepherd ND, D'alessandro DM. Structurally photo-active metal-organic frameworks: Incorporation methods, response tuning, and potential applications. Chem Phys Rev 2021;2:011301. DOI

141. Gangu KK, Maddila S, Mukkamala SB, Jonnalagadda SB. A review on contemporary metal-organic framework materials. Inorganica Chimica Acta 2016;446:61-74. DOI 
142. Fang Z, Bueken B, De Vos DE, Fischer RA. Defect-engineered metal-organic frameworks. Angew Chem Int Ed Engl 2015;54:723454. DOI PubMed PMC

143. Dissegna S, Epp K, Heinz WR, Kieslich G, Fischer RA. Defective metal-organic frameworks. Adv Mater 2018;30:e1704501. DOI PubMed

144. Zhou Z, Zhang Q, Sun J, et al. Metal-organic framework derived spindle-like carbon incorporated $\alpha-\mathrm{Fe}_{2} \mathrm{O}_{3}$ grown on carbon nanotube fiber as anodes for high-performance wearable asymmetric supercapacitors. ACS Nano 2018;12:9333-41. DOI PubMed

145. Zhou J, Yuan Y, Tang J, Tang W. Metal-organic frameworks governed well-aligned conducting polymer/bacterial cellulose membranes with high areal capacitance. Energy Storage Materials 2019;23:594-601. DOI

146. Yang J, Xiong P, Zheng C, Qiu H, Wei M. Metal-organic frameworks: a new promising class of materials for a high performance supercapacitor electrode. J Mater Chem A 2014;2:16640-4. DOI

147. Wang K, Bi R, Huang M, et al. Porous cobalt metal-organic frameworks as active elements in battery-supercapacitor hybrid devices. Inorg Chem 2020;59:6808-14. DOI PubMed

148. Liu Y, Xu X, Shao Z, Jiang SP. Metal-organic frameworks derived porous carbon, metal oxides and metal sulfides-based compounds for supercapacitors application. Energy Storage Materials 2020;26:1-22. DOI

149. Wang K, Xun Q, Zhang Q. Recent progress in metal-organic frameworks as active materials for supercapacitors. EnergyChem 2020;2:100025. DOI

150. Cherusseri J, Pandey D, Sambath Kumar K, Thomas J, Zhai L. Flexible supercapacitor electrodes using metal-organic frameworks. Nanoscale 2020;12:17649-62. DOI PubMed

151. Xu X, Tang J, Qian H, et al. Three-dimensional networked metal-organic frameworks with conductive polypyrrole tubes for flexible supercapacitors. ACS Appl Mater Interfaces 2017;9:38737-44. DOI PubMed

152. Wang B, Liu S, Liu L, et al. MOF/PEDOT/HPMo-based polycomponent hierarchical hollow micro-vesicles for high performance flexible supercapacitors. J Mater Chem A 2021;9:2948-58. DOI

153. Xie LS, Skorupskii G, Dincă M. Electrically conductive metal-organic frameworks. Chem Rev 2020;120:8536-80. DOI PubMed PMC

154. Hou R, Miao M, Wang Q, et al. Integrated conductive hybrid architecture of metal - organic framework nanowire array on polypyrrole membrane for all-solid-state flexible supercapacitors. Adv Energy Mater 2019;10:1901892. DOI

155. Zhang W, Li R, Zheng H, Bao J, Tang Y, Zhou K. Laser-assisted printing of electrodes using metal - organic frameworks for microsupercapacitors. Adv Funct Mater 2021;31:2009057. DOI

156. Naguib M, Kurtoglu M, Presser V, et al. Two-dimensional nanocrystals produced by exfoliation of Ti3 AlC2. Adv Mater 2011;23:4248-53. DOI PubMed

157. Wang L, Zhang M, Yang B, Tan J, Ding X, Li W. Recent advances in multidimensional (1D, 2D, and 3D) composite sensors derived from MXene: synthesis, structure, application, and perspective. Small Methods 2021;5:2100409. DOI

158. Hu M, Zhang H, Hu T, Fan B, Wang X, Li Z. Emerging 2D MXenes for supercapacitors: status, challenges and prospects. Chem Soc Rev 2020;49:6666-93. DOI PubMed

159. Deysher G, Shuck CE, Hantanasirisakul K, et al. Synthesis of $\mathrm{Mo}_{4} \mathrm{VAlC}_{4} \mathrm{MAX}$ phase and two-dimensional $\mathrm{Mo}_{4} \mathrm{VC}_{4} \mathrm{MXene}$ with five atomic layers of transition metals. ACS Nano 2020;14:204-17. DOI PubMed

160. Ibrahim Y, Mohamed A, Abdelgawad AM, Eid K, Abdullah AM, Elzatahry A. The recent advances in the mechanical properties of self-standing two-dimensional MXene-based nanostructures: deep insights into the supercapacitor. Nanomaterials (Basel) 2020;10:1916. DOI PubMed PMC

161. Lei J, Zhang X, Zhou Z. Recent advances in MXene: preparation, properties, and applications. Front Phys 2015;10:276-86. DOI

162. Shuck CE, Sarycheva A, Anayee M, et al. Scalable synthesis of $\mathrm{Ti}_{3} \mathrm{C}_{2} \mathrm{~T}_{\mathrm{x}} \mathrm{MXene}$. Adv Eng Mater 2020;22:1901241. DOI

163. Zhan X, Si C, Zhou J, Sun Z. MXene and MXene-based composites: synthesis, properties and environment-related applications. Nanoscale Horiz 2020;5:235-58. DOI

164. Shen S, Ke T, Rajavel K, Yang K, Lin D. Dispersibility and photochemical stability of delaminated MXene flakes in water. Small 2020;16:e2002433. DOI PubMed

165. Akuzum B, Maleski K, Anasori B, et al. Rheological characteristics of 2D titanium carbide (MXene) dispersions: a guide for processing MXenes. ACS Nano 2018;12:2685-94. DOI PubMed

166. Li Y, Lu Z, Xin B, Liu Y, Cui Y, Hu Y. All-solid-state flexible supercapacitor of carbonized MXene/Cotton fabric for wearable energy storage. Appl Surf Sci 2020;528:146975. DOI

167. Hu M, Li Z, Li G, Hu T, Zhang C, Wang X. All-solid-state flexible fiber-based mxene supercapacitors. Adv Mater Technol 2017;2:1700143. DOI

168. Yu C, Gong Y, Chen R, et al. A solid-state fibriform supercapacitor boosted by host-guest hybridization between the carbon nanotube scaffold and MXene nanosheets. Small 2018:e1801203. DOI PubMed

169. Li H, Chen R, Ali M, Lee H, Ko MJ. In Situ Grown MWCNTs/MXenes Nanocomposites on carbon cloth for high-performance flexible supercapacitors. Adv Funct Mater 2020;30:2002739. DOI

170. Ling Z, Ren CE, Zhao MQ, et al. Flexible and conductive MXene films and nanocomposites with high capacitance. Proc Natl Acad Sci U S A 2014;111:16676-81. DOI PubMed PMC

171. Hasan MM, Hossain MM, Chowdhury HK. Two-dimensional MXene-based flexible nanostructures for functional nanodevices: a review. J Mater Chem A 2021;9:3231-69. DOI

172. Zhang CJ, McKeon L, Kremer MP, et al. Additive-free MXene inks and direct printing of micro-supercapacitors. Nat Commun 2019;10:1795. DOI PubMed PMC 
173. Wen D, Wang X, Liu L, et al. Inkjet printing transparent and conductive MXene $\left(\mathrm{Ti}_{3} \mathrm{C}_{2} \mathrm{~T}_{\mathrm{x}}\right)$ films: a strategy for flexible energy storage devices. ACS Appl Mater Interfaces 2021;13:17766-80. DOI PubMed

174. Zhu M, Huang Y, Deng Q, et al. Highly flexible, freestanding supercapacitor electrode with enhanced performance obtained by hybridizing polypyrrole chains with MXene. Adv Energy Mater 2016;6:1600969. DOI

175. Qin L, Tao Q, El Ghazaly A, et al. High-performance ultrathin flexible solid-state supercapacitors based on solution processable $\mathrm{Mo}_{1.33} \mathrm{C}$ MXene and PEDOT:PSS. Adv Funct Mater 2018;28:1703808. DOI

176. Zhou T, Wu C, Wang Y, et al. Super-tough MXene-functionalized graphene sheets. Nat Commun 2020;11:2077. DOI PubMed PMC

177. Wang R, Luo S, Xiao C, et al. MXene-carbon nanotubes layer-by-layer assembly based on-chip micro-supercapacitor with improved capacitive performance. Electrochimica Acta 2021;386:138420. DOI

178. Yu L, Hu L, Anasori B, et al. MXene-bonded activated carbon as a flexible electrode for high-performance supercapacitors. ACS Energy Lett 2018;3:1597-603. DOI

179. Zheng X, Shen J, Hu Q, et al. Vapor phase polymerized conducting polymer/MXene textiles for wearable electronics. Nanoscale 2021;13:1832-41. DOI PubMed

180. Ma Y, Sheng H, Dou W, et al. $\mathrm{Fe}_{2} \mathrm{O}_{3}$ nanoparticles anchored on the $\mathrm{Ti}_{3} \mathrm{C}_{2} \mathrm{~T}_{\mathrm{x}}$ MXene paper for flexible supercapacitors with ultrahigh volumetric capacitance. ACS Appl Mater Interfaces 2020;12:41410-8. DOI PubMed

181. Patil AM, Kitiphatpiboon N, An X, et al. Fabrication of a high-energy flexible all-solid-state supercapacitor using pseudocapacitive 2D-Ti $\mathrm{C}_{3} \mathrm{C}_{2}$-MXene and battery-type reduced graphene oxide/nickel-cobalt bimetal oxide electrode materials. ACS Appl Mater Interfaces 2020;12:52749-62. DOI PubMed

182. Xie W, Wang Y, Zhou J, et al. MOF-derived CoFe2O4 nanorods anchored in MXene nanosheets for all pseudocapacitive flexible supercapacitors with superior energy storage. Applied Surface Science 2020;534:147584. DOI

183. Choudhury NA, Sampath S, Shukla AK. Hydrogel-polymer electrolytes for electrochemical capacitors: an overview. Energy Environ Sci 2009;2:55-67. DOI

184. Huang Y, Zhong M, Shi F, et al. An intrinsically stretchable and compressible supercapacitor containing a polyacrylamide hydrogel electrolyte. Angew Chem Int Ed Engl 2017;56:9141-5. DOI PubMed

185. Han L, Huang H, Fu X, et al. A flexible, high-voltage and safe zwitterionic natural polymer hydrogel electrolyte for high-energydensity zinc-ion hybrid supercapacitor. Chem Eng J 2020;392:123733. DOI

186. Shi X, Wu ZS, Qin J, et al. Graphene-based linear tandem micro-supercapacitors with metal-free current collectors and high-voltage output. Adv Mater 2017;29:1703034. DOI PubMed

187. Wu ZS, Liu Z, Parvez K, Feng X, Müllen K. Ultrathin printable graphene supercapacitors with AC line-filtering performance. $A d v$ Mater 2015;27:3669-75. DOI PubMed

188. Xiong G, He P, Huang B, Chen T, Bo Z, Fisher TS. Graphene nanopetal wire supercapacitors with high energy density and thermal durability. Nano Energy 2017;38:127-36. DOI

189. Nguyen PT, Jang J, Lee Y, Choi ST, In JB. Laser-assisted fabrication of flexible monofilament fiber supercapacitors. J Mater Chem A 2021;9:4841-50. DOI

190. Zhang X, Fu Q, Huang H, Wei L, Guo X. Silver-quantum-dot-modified $\mathrm{MoO}_{3}$ and $\mathrm{MnO}_{2}$ paper-like freestanding films for flexible solid-state asymmetric supercapacitors. Small 2019;15:e1805235. DOI PubMed

191. Kim JW, Park H, Lee G, et al. Paper-like, thin, foldable, and self-healable electronics based on PVA/CNC nanocomposite film. $A d v$ Funct Mater 2019;29:1905968. DOI

192. Liu Z, Zhang J, Liu J, et al. Highly compressible and superior low temperature tolerant supercapacitors based on dual chemically crosslinked PVA hydrogel electrolytes. J Mater Chem A 2020;8:6219-28. DOI

193. Peng H, Lv Y, Wei G, et al. A flexible and self-healing hydrogel electrolyte for smart supercapacitor. J Power Sources 2019;431:2109. DOI

194. Alipoori S, Mazinani S, Aboutalebi SH, Sharif F. Review of PVA-based gel polymer electrolytes in flexible solid-state supercapacitors: opportunities and challenges. J Energy Storage 2020;27:101072. DOI

195. Wei JS, Ding C, Zhang P, et al. Robust negative electrode materials derived from carbon dots and porous hydrogels for highperformance hybrid supercapacitors. Adv Mater 2019;31:e1806197. DOI PubMed

196. Zhao C, Wang C, Yue Z, Shu K, Wallace GG. Intrinsically stretchable supercapacitors composed of polypyrrole electrodes and highly stretchable gel electrolyte. ACS Appl Mater Interfaces 2013;5:9008-14. DOI PubMed

197. Fang C, Zhang D. A large areal capacitance structural supercapacitor with a 3D rGO@ $\mathrm{MnO}_{2}$ foam electrode and polyacrylic acidPortland cement - KOH electrolyte. J Mater Chem A 2020;8:12586-93. DOI

198. Cevik E, Bozkurt A. Redox active polymer metal chelates for use in flexible symmetrical supercapacitors: cobalt-containing poly(acrylic acid) polymer electrolytes. J Energy Chem 2021;55:145-53. DOI

199. Huang Y, Zhong M, Huang Y, et al. A self-healable and highly stretchable supercapacitor based on a dual crosslinked polyelectrolyte. Nat Commun 2015;6:10310. DOI PubMed PMC

200. Choudhury NA, Sampath S, Shukla AK. Gelatin hydrogel electrolytes and their application to electrochemical supercapacitors. $J$ Electrochem Soc 2008;155:A74. DOI

201. Yun TG, Jang J, Cheong JY, Kim I. Organism epidermis/plant-root inspired ultra-stable supercapacitor for large-scale wearable energy storage applications. Nano Energy 2021;82:105776. DOI

202. Liu J, Song H, Wang Z, Zhang J, Zhang J, Ba X. Stretchable, self-healable, and reprocessable chemical cross-linked ionogels electrolytes based on gelatin for flexible supercapacitors. J Mater Sci 2020;55:3991-4004. DOI 
203. Park JH, Rana HH, Lee JY, Park HS. Renewable flexible supercapacitors based on all-lignin-based hydrogel electrolytes and nanofiber electrodes. J Mater Chem A 2019;7:16962-8. DOI

204. Peng Z, Zou Y, Xu S, Zhong W, Yang W. High-performance biomass-based flexible solid-state supercapacitor constructed of pressure-sensitive lignin-based and cellulose hydrogels. ACS Appl Mater Interfaces 2018;10:22190-200. DOI PubMed

205. Yang L, Song L, Feng Y, et al. Zinc ion trapping in a cellulose hydrogel as a solid electrolyte for a safe and flexible supercapacitor. $J$ Mater Chem A 2020;8:12314-8. DOI

206. Li X, Yuan L, Liu R, et al. Engineering textile electrode and bacterial cellulose nanofiber reinforced hydrogel electrolyte to enable high-performance flexible all-solid-state supercapacitors. Adv Energy Mater 2021;11:2003010. DOI

207. Wang C, Yokota T, Someya T. Natural biopolymer-based biocompatible conductors for stretchable bioelectronics. Chem Rev 2021;121:2109-46. DOI PubMed

208. Chen M, Chen J, Zhou W, Han X, Yao Y, Wong CP. Realizing an all-round hydrogel electrolyte toward environmentally adaptive dendrite-free aqueous $\mathrm{Zn}-\mathrm{MnO}_{2}$ batteries. Adv Mater 2021;33:e2007559. DOI PubMed

209. Huang H, Han L, Fu X, et al. A powder self-healable hydrogel electrolyte for flexible hybrid supercapacitors with high energy density and sustainability. Small 2021;17:e2006807. DOI PubMed

210. Wang J, Liu F, Tao F, Pan Q. Rationally designed self-healing hydrogel electrolyte toward a smart and sustainable supercapacitor. ACS Appl Mater Interfaces 2017;9:27745-53. DOI PubMed

211. Tao F, Qin L, Wang Z, Pan Q. Self-healable and cold-resistant supercapacitor based on a multifunctional hydrogel electrolyte. ACS Appl Mater Interfaces 2017;9:15541-8. DOI PubMed

212. Yu H, Rouelle N, Qiu A, et al. Hydrogen bonding-reinforced hydrogel electrolyte for flexible, robust, and all-in-one supercapacitor with excellent low-temperature tolerance. ACS Appl Mater Interfaces 2020;12:37977-85. DOI PubMed

213. Yang P, Feng C, Liu Y, et al. Thermal self-protection of zinc-ion batteries enabled by smart hygroscopic hydrogel electrolytes. $A d v$ Energy Mater 2020;10:2002898. DOI

214. Peng H, Gao X, Sun K, et al. Physically cross-linked dual-network hydrogel electrolyte with high self-healing behavior and mechanical strength for wide-temperature tolerant flexible supercapacitor. Chemical Engineering Journal 2021;422:130353. DOI

215. Yin B, Zhang S, Ke K, Wang Z. Advanced deformable all-in-one hydrogel supercapacitor based on conducting polymer: toward integrated mechanical and capacitive performance. Journal of Alloys and Compounds 2019;805:1044-51. DOI

216. Guo L, Ma W, Wang Y, et al. A chemically crosslinked hydrogel electrolyte based all-in-one flexible supercapacitor with superior performance. J Alloys Compd 2020;843:155895. DOI

217. Guo Y, Zheng K, Wan P. A flexible stretchable hydrogel electrolyte for healable all-in-one configured supercapacitors. Small 2018;14:e1704497. DOI PubMed

218. Hu M, Wang J, Liu J, Zhang J, Ma X, Huang Y. An intrinsically compressible and stretchable all-in-one configured supercapacitor. Chem Commun (Camb) 2018;54:6200-3. DOI PubMed

219. Wang K, Zhang X, Li C, et al. Chemically crosslinked hydrogel film leads to integrated flexible supercapacitors with superior performance. Adv Mater 2015;27:7451-7. DOI PubMed

220. Jin X, Song L, Yang H, et al. Stretchable supercapacitor at -30 ${ }^{\circ}$ C. Energy Environ Sci 2021;14:3075-85. DOI

221. Hsu HH, Liu Y, Wang Y, et al. Mussel-inspired autonomously self-healable all-in-one supercapacitor with biocompatible hydrogel. ACS Sustainable Chem Eng 2020;8:6935-48. DOI

222. Zeng J, Dong L, Sha W, Wei L, Guo X. Highly stretchable, compressible and arbitrarily deformable all-hydrogel soft supercapacitors. Chem Eng J 2020;383:123098. DOI

223. Han J, Wang H, Yue Y, et al. A self-healable and highly flexible supercapacitor integrated by dynamically cross-linked electroconductive hydrogels based on nanocellulose-templated carbon nanotubes embedded in a viscoelastic polymer network. Carbon 2019;149:1-18. DOI

224. Wen N, Jiang B, Wang X, et al. Overview of polyvinyl alcohol nanocomposite hydrogels for electro-skin, actuator, supercapacitor and fuel cell. Chem Rec 2020;20:773-92. DOI PubMed

225. Hua M, Wu S, Ma Y, et al. Strong tough hydrogels via the synergy of freeze-casting and salting out. Nature 2021;590:594-9. DOI PubMed

226. Ben J, Song Z, Liu X, Lü W, Li X. Fabrication and electrochemical performance of PVA/CNT/PANI flexible films as electrodes for supercapacitors. Nanoscale Res Lett 2020;15:151. DOI PubMed PMC

227. Jia Z, Gong J, Zeng Y, et al. Bioinspired conductive silk microfiber integrated bioelectronic for diagnosis and wound healing in diabetes. Adv Funct Mater 2021;31:2010461. DOI

228. Jia Z, Lv X, Hou Y, et al. Mussel-inspired nanozyme catalyzed conductive and self-setting hydrogel for adhesive and antibacterial bioelectronics. Bioact Mater 2021;6:2676-87. DOI PubMed PMC

229. Gan D, Shuai T, Wang X, et al. Mussel-inspired redox-active and hydrophilic conductive polymer nanoparticles for adhesive hydrogel bioelectronics. Nanomicro Lett 2020;12:169. DOI PubMed PMC

230. Han L, Liu K, Wang M, et al. Mussel-inspired adhesive and conductive hydrogel with long-lasting moisture and extreme temperature tolerance. Adv Funct Mater 2018;28:1704195. DOI

231. Gan D, Huang Z, Wang X, et al. Graphene oxide-templated conductive and redox-active nanosheets incorporated hydrogels for adhesive bioelectronics. Adv Funct Mater 2019;30:1907678. DOI

232. Han L, Lu X, Wang M, et al. A mussel-inspired conductive, self-adhesive, and self-healable tough hydrogel as cell stimulators and implantable bioelectronics. Small 2017;13:1601916. DOI PubMed

233. Zhang C, Wu B, Zhou Y, Zhou F, Liu W, Wang Z. Mussel-inspired hydrogels: from design principles to promising applications. 
Chem Soc Rev 2020;49:3605-37. DOI PubMed

234. Xie C, Wang X, He H, Ding Y, Lu X. Mussel-inspired hydrogels for self-adhesive bioelectronics. Adv Funct Mater 2020;30:1909954. DOI

235. Son EJ, Kim JH, Kim K, Park CB. Quinone and its derivatives for energy harvesting and storage materials. J Mater Chem A 2016;4:11179-202. DOI

236. Han C, Li H, Shi R, et al. Organic quinones towards advanced electrochemical energy storage: recent advances and challenges. $J$ Mater Chem A 2019;7:23378-415. DOI

237. Katsuyama Y, Nakayasu Y, Oizumi K, Fujihara Y, Kobayashi H, Honma I. Quinone-based redox supercapacitor using highly conductive hard carbon derived from oak wood. Adv Sustainable Syst 2019;3:1900083. DOI

238. Sumboja A, Foo CY, Wang X, Lee PS. Large areal mass, flexible and free-standing reduced graphene oxide/manganese dioxide paper for asymmetric supercapacitor device. Adv Mater 2013;25:2809-15. DOI PubMed

239. Wang S, Liu N, Su J, et al. Highly stretchable and self-healable supercapacitor with reduced graphene oxide based fiber springs. ACS Nano 2017;11:2066-74. DOI PubMed

240. Yang JC, Mun J, Kwon SY, Park S, Bao Z, Park S. Electronic skin: recent progress and future prospects for skin-attachable devices for health monitoring, robotics, and prosthetics. Adv Mater 2019;31:e1904765. DOI PubMed

241. Lee Y, Myoung J, Cho S, et al. Bioinspired gradient conductivity and stiffness for ultrasensitive electronic skins. ACS Nano 2021;15:1795-804. DOI PubMed

242. Hua Q, Sun J, Liu H, et al. Skin-inspired highly stretchable and conformable matrix networks for multifunctional sensing. Nat Commun 2018;9:244. DOI PubMed PMC

243. Liu Z, Li H, Shi B, Fan Y, Wang ZL, Li Z. Wearable and implantable triboelectric nanogenerators. Adv Funct Mater 2019;29:1808820. DOI

244. Liu W, Wang Z, Wang G, et al. Integrated charge excitation triboelectric nanogenerator. Nat Commun 2019;10:1426. DOI PubMed PMC

245. Zang X, Zhang R, Zhen Z, et al. Flexible, temperature-tolerant supercapacitor based on hybrid carbon film electrodes. Nano Energy 2017;40:224-32. DOI

246. Rong Q, Lei W, Huang J, Liu M. Low temperature tolerant organohydrogel electrolytes for flexible solid-state supercapacitors. $A d v$ Energy Mater 2018;8:1801967. DOI 